\title{
Two-stream instabilities from the lower-hybrid frequency to the electron cyclotron frequency: application to the front of quasi-perpendicular shocks
}

\author{
Laurent Muschietti ${ }^{1,2}$ and Bertrand Lembège ${ }^{1}$ \\ ${ }^{1}$ LATMOS-IPSL-UVSQ-CNRS, Guyancourt, 78280, France \\ ${ }^{2}$ SSL, UCB, Berkeley, CA 94720, USA \\ Correspondence to: Laurent Muschietti (laurent@ ssl.berkeley.edu)
}

Received: 27 April 2017 - Revised: 11 July 2017 - Accepted: 19 July 2017 - Published: 15 September 2017

\begin{abstract}
Quasi-perpendicular supercritical shocks are characterized by the presence of a magnetic foot due to the accumulation of a fraction of the incoming ions that is reflected by the shock front. There, three different plasma populations coexist (incoming ion core, reflected ion beam, electrons) and can excite various two-stream instabilities (TSIs) owing to their relative drifts. These instabilities represent local sources of turbulence with a wide frequency range extending from the lower hybrid to the electron cyclotron. Their linear features are analyzed by means of both a dispersion study and numerical PIC simulations. Three main types of TSI and correspondingly excited waves are identified:
\end{abstract}

i. Oblique whistlers due to the (so-called "fast") relative drift between reflected ions/electrons; the waves propagate toward upstream away from the shock front at a strongly oblique angle $\left(\theta \sim 50^{\circ}\right)$ to the ambient magnetic field $\boldsymbol{B}_{o}$, have frequencies a few times the lower hybrid, and have wavelengths a fraction of the ion inertia length $c / \omega_{\mathrm{pi}}$.

ii. Quasi-perpendicular whistlers due to the (so-called "slow") relative drift between incoming ions/electrons; the waves propagate toward the shock ramp at an angle $\theta$ a few degrees off $90^{\circ}$, have frequencies around the lower hybrid, and have wavelengths several times the electron inertia length $c / \omega_{\text {pe }}$.

iii. Extended Bernstein waves which also propagate in the quasi-perpendicular domain, yet are due to the (so-called "fast") relative drift between reflected ions/electrons; the instability is an extension of the electron cyclotron drift instability (normally strictly perpen- dicular and electrostatic) and produces waves with a magnetic component which have frequencies close to the electron cyclotron as well as wavelengths close to the electron gyroradius and which propagate toward upstream.

Present results are compared with previous works in order to stress some features not previously analyzed and to define a more synthetic view of these TSIs.

Keywords. Interplanetary physics (planetary bow shocks) magnetospheric physics (plasma waves and instabilities) space plasma physics (wave-particle interactions)

\section{Introduction}

A hallmark of supercritical shocks in collisionless plasmas is the presence of a sizable ion population that is reflected off of the steep shock front. These ions carry a substantial amount of energy: they are the source of microturbulence within the shock front and are fundamental to the transformation of directed bulk flow energy into thermal energy, a tenet of shock physics. For quasi-perpendicular geometries, the reflected ions' velocity, as seen in the normal incidence frame, is in large part directed at $90^{\circ}$ to the magnetic field $\boldsymbol{B}_{o}$. The relative drifts across $\boldsymbol{B}_{o}$ between the populations of incoming ions, reflected ions, and electrons enable the excitation of several microinstabilities (Wu et al., 1984, and references within)

Whistler waves are an attribute of collisionless fast-mode shocks. They have been observed in association with shocks 
in space for a very long time (e.g., Rodriguez and Gurnett, 1975). The term "whistler" covers waves over a large range of frequencies and many observations related to shocks pertain to the ion frequency range (a few hertz and below). Waves with higher frequencies from the lower-hybrid to the electron cyclotron range have also been observed. Their characteristics, however, can be difficult to establish because of a potentially important Doppler shift in frequency between the spacecraft frame where they are measured and the plasma frame where they can be properly identified. Whistler waves especially in the lower-hybrid range have interested theorists and simulationists owing to their potential role for transferring energy between ions and electrons (e.g., Wu et al., 1983; Winske et al., 1985; Matsukiyo and Scholer, 2003, 2006). Observationally, an important characteristic of whistlers in this regime is that the waves appear to propagate obliquely with respect to $\boldsymbol{B}_{o}$ (Krasnoselskikh et al., 1991; Hull et al., 2012; Sundkvist et al., 2012; Dimmock et al., 2013). Moreover, when the waves can be put into their macroscopic context, their wavevectors have been measured as equally oblique with respect to the shock normal (Hull et al., 2012; Dimmock et al., 2013). Since the normal presumably corresponds to the direction of the drift between the ion populations, the waves appear to propagate at a sizable angle with respect to the drift. The measurements made by the Polar mission, which recorded a substantial number of whistler waves as detailed by Hull et al. (2012), benefit from capturing all components of the electric and magnetic fields. The whistler waves in the lower-hybrid frequency range, it was concluded, have wavevectors which are close to the coplanarity plane and which make an angle $\sim 50^{\circ}$ to $\boldsymbol{B}_{o}$ and $\sim 50^{\circ}$ to the shock normal (where the latter is pointing upstream).

In this article, we present a synthetic view of the plasma microinstabilities which can occur in the foot of supercritical quasi-perpendicular shocks as the result of the relative drifts between incoming ions, reflected ions, and electrons. Figure 1 illustrates the relations between the three plasma populations in the shock's foot. The resulting instabilities cover wavelengths from the ion inertia length to the electron gyroradius and frequencies from the lower-hybrid to the electron cyclotron. The study can be viewed as an extension of our previous work, which was focussed on $90^{\circ}$ propagation and electron Bernstein waves (Muschietti and Lembège, 2013). By contrast, we consider here various propagation angles and lower frequencies, with a special emphasis on whistlers that propagate obliquely and have frequencies in the lower-hybrid range.

Our notations are as follows: $V_{\mathrm{A}}$ designates the Alfvén speed, $c$ is the speed of light, $\omega_{\mathrm{pi}}\left(\omega_{\mathrm{pe}}\right)$ is the ion (electron) plasma frequency, and $\Omega_{\mathrm{ci}}\left(\Omega_{\mathrm{ce}}\right)$ is the ion (electron) cyclotron frequency. When we use the warm plasma model, $T_{j}$ (where $j=\mathrm{e}, \mathrm{c}, \mathrm{b}$ ) represents the temperature of the electrons (subscript e), the incoming ion core (subscript c), and the reflected ion beam (subscript b), respectively. The associated betas (ratio of thermal pressure to magnetic pressure)

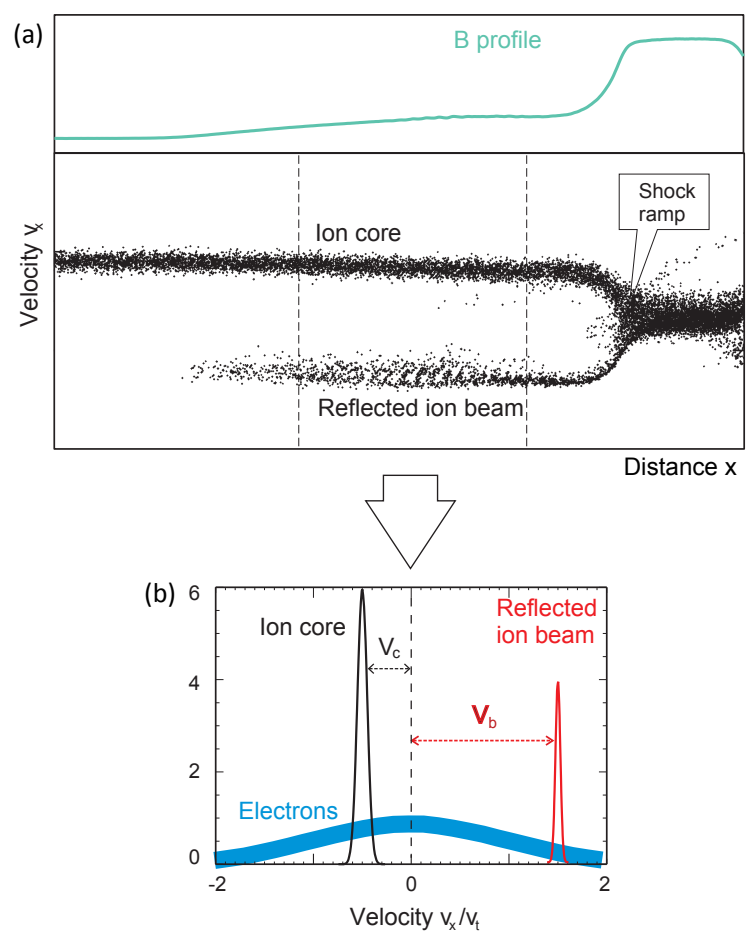

Figure 1. Model of ion and electron populations in the foot region of a supercritical perpendicular shock extracted at a given time from a 1-D PIC simulation. (a) View of the ion phase space with profile of the magnetic field $\boldsymbol{B}$; (b) enlargement of the local ion core, reflected ion beam, and electron distributions to be used for the dispersion analysis; reference frame set such that the electrons are at rest.

are defined as $\beta_{j}=\left(8 \pi n_{j} T_{j}\right) / B_{o}{ }^{2}$, where the densities $n_{j}$ satisfies $n_{\mathrm{e}}=n_{\mathrm{c}}+n_{\mathrm{b}}=n_{\mathrm{i}}$. Finally $M_{\mathrm{i}}\left(m_{\mathrm{e}}\right)$ denotes the ion (electron) mass.

Results of linear dispersion analysis are presented in Sect. 2 for a stable situation without beam. We first address the cold plasma model in Sect. 2.1. In Sect. 2.2 we show that the electrons are in a kinetic regime and that thermal effects are very important, unless an extremely small $\beta_{\mathrm{e}}$ is assumed. We treat the unstable case where there is an ion drift in Sect. 3. Again, we examine the question first within the framework of the cold model (Sect. 3.1), then turn to the warm plasma by numerically solving the full dispersion relation (Sect. 3.2). The extension of the electron cyclotron drift instability (ECDI) beyond the electrostatic framework and to quasi-perpendicular angles (close to yet off $90^{\circ}$ ) is discussed in Sect. 4. In Sect. 5 we present PIC simulations to illustrate the dispersion results of Sects. 2-4. Finally, Sect. 6 discusses our results and Sect. 7 concludes our work. 


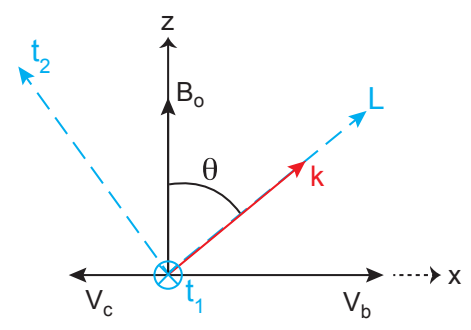

Figure 2. Orientation of the wavevector $\boldsymbol{k}$ with respect to the directions of the background magnetic field $\left(0,0, B_{o}\right)$ and beam $\left(V_{\mathrm{b}}, 0,0\right)$. In blue are axes $t_{1}$ (into the page), $t_{2}$, and $L$ (along vector $\boldsymbol{k}$ ) used in 1-D oblique simulations, which are performed with a predetermined angle $\theta$ defined from the dispersion study.

\section{Whistler mode in oblique propagation (without ion beam)}

\subsection{Cold approximation}

In the cold plasma model, the mode which can propagate in the frequency range above the ion cyclotron frequency is the right-handed wave. It is often referred to as the R-X mode because it becomes the extraordinary wave in perpendicular propagation (e.g., Swanson, 2003). Let $\theta$ be the angle between the wavevector and the direction of the background magnetic field $\boldsymbol{B}_{o}=\left(0,0, B_{o}\right)$, as displayed in Fig. 2. A convenient, approximate expression for its dispersion relation can be obtained from the low-frequency relation that Stringer (1963) derived using fluid equations and neglecting terms of order $m_{\mathrm{e}} / M_{\mathrm{i}}$. Assuming further that the phase speed is much larger than the acoustic speed, one can write the explicit dispersion relation

$$
\begin{aligned}
\omega(k, \theta) & =\frac{k V_{\mathrm{A}}}{\left[1+\left(k c / \omega_{\mathrm{pe}}\right)^{2}\right]^{1 / 2}} \\
& \times\left[1+\cos ^{2} \theta \frac{\left(k c / \omega_{\mathrm{pi}}\right)^{2}}{1+\left(k c / \omega_{\mathrm{pe}}\right)^{2}}\right]^{1 / 2} .
\end{aligned}
$$

In the very low-frequency $\left(\omega \ll \Omega_{\mathrm{ci}}\right)$ and long wavelength limit $\left(k c / \omega_{\text {pi }} \ll 1\right)$, we recover a magnetosonic wave with phase velocity equal to the Alfvén speed $V_{\mathrm{A}}$. When frequencies become comparable to the ion cyclotron frequency, i.e., $k V_{\mathrm{A}} \sim \Omega_{\mathrm{ci}}$, the term $k c / \omega_{\mathrm{pi}}=k V_{\mathrm{A}} / \Omega_{\mathrm{ci}}$ becomes of order unity, whereby an angle $\theta$ dependence appears. As the wavenumber keeps increasing, $k c / \omega_{\mathrm{pi}}>1$, the second term in the square bracket becomes important, the phase speed increases, and the mode enters the domain of the whistler wave.

Figure 3 shows the dispersion relation in a log-log representation for different angles $\theta$. Colors are used to distinguish between three angles: quasi-parallel propagation with $10^{\circ}$ (green), oblique with $55^{\circ}$ (blue), and quasi-perpendicular with $85^{\circ}$ (red). The wavenumbers $k$ (horizontal axis) range from $\omega_{\mathrm{pi}} / c$ to many $\omega_{\mathrm{pe}} / c$ and the frequencies $\omega$ (vertical

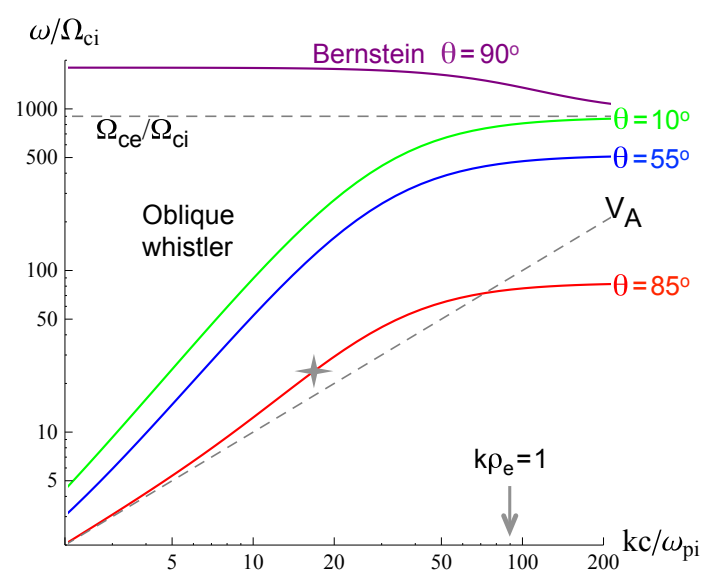

Figure 3. Solutions of the cold dispersion relation (without ion beam) above the ion cyclotron frequency $\Omega_{\text {ci }}$ in a log-log representation, shown for three propagation angles for the right-handed, whistler wave (from Eq. 1). Grey, dashed lines indicate the electron cyclotron frequency $\Omega_{\mathrm{ce}}$ and the phase speed $V_{\mathrm{A}}$. A grey star on the $\theta=85^{\circ}$ solution marks the location where the dispersion curve changes from concave to convex. For reference, electron temperature effects (see Table 1) are introduced to indicate the Bernstein branch defined for $90^{\circ}$ propagation and the electron gyroradius scale $\left(k \rho_{\mathrm{e}}=1\right)$.

axis) range from $\Omega_{\mathrm{ci}}$ up to $\Omega_{\mathrm{ce}}$, which is marked here with a horizontal dashed line. The sloped dashed line indicates the Alfvén speed. If $\left(\cos \theta k c / \omega_{\mathrm{pi}}\right)^{2} \gg 1$, the frequency $\omega$ increases quadratically with $k$ and one can rewrite Eq. (1) into the familiar whistler relation

$\omega=\Omega_{\mathrm{ce}} \cos \theta \frac{\left(k c / \omega_{\mathrm{pe}}\right)^{2}}{1+\left(k c / \omega_{\mathrm{pe}}\right)^{2}}$.

For very short wavelengths, $k \gg \omega_{\text {pe }} / c$, the dispersion presents a resonance, becomes independent of $k$, and simplifies to $\omega=\Omega_{\mathrm{ce}} \cos \theta$. Noteworthy is the double curvature of $\omega(k)$, first concave (at low $k$ ) and then convex (at high $k$ ) (e.g., Swanson, 2003, Fig. 3.1) (e.g., Tidman and Krall, 1971, Fig. 2.3). As visible here in Fig. 3 for $\theta=85^{\circ}$, the dispersion changes from concave to convex at the point marked with a star. The feature plays an important role as we will see when discussing the effect of a beam in Sect. 3 .

For drawing Fig. 3, we choose parameter values such as mass ratio and magnetization that are the same as those used in the simulations to be shown later, namely $M / m=900$ and $\omega_{\text {pe }} / \Omega_{\mathrm{ce}}=10$. This will facilitate future comparisons. In addition, we refer the reader to Table 1 and assume a value of $\beta_{\mathrm{e}}=0.22$ to mark the position of the electron gyroradius as well as the first Bernstein branch on the plot. 
Table 1. Plasma parameters in normalized units.

\begin{tabular}{ll}
\hline Alfvén speed & $V_{\mathrm{A}}=1$ \\
Ion inertia length & $c / \omega_{\mathrm{pi}}=1$ \\
Ion gyrofrequency & $\Omega_{\mathrm{ci}}=1$ \\
Speed of light & $c=300$ \\
Ion/electron mass & $M_{\mathrm{i}} / m_{\mathrm{e}}=900$ \\
Plasma/cyclotron & \\
Frequency & $\omega_{\mathrm{pe}} / \Omega_{\mathrm{ce}}=10$ \\
Electron beta & $\beta_{\mathrm{e}}=0.22$ \\
Ion beta & $\beta_{\mathrm{i}}=0.22$ \\
\hline
\end{tabular}

\subsection{Thermal effects on obliquely propagating whistlers}

Ion temperature effects are negligible. Indeed, since $v_{\mathrm{ti}}=$ $V_{\mathrm{A}}\left(\beta_{\mathrm{i}} / 2\right)^{1 / 2}$, where $v_{\mathrm{ti}} \equiv \sqrt{T_{\mathrm{i}} / M_{\mathrm{i}}}$ is the thermal velocity of the ions, one can substitute $v_{\mathrm{ti}}$ for $V_{\mathrm{A}}$ in Eq. (1). It is easy then to see that $k v_{\mathrm{ti}} / \omega<\left(\beta_{\mathrm{i}} / 2\right)^{1 / 2}$, unless one deals with very short wavelengths such that $k c / \omega_{\mathrm{pe}} \gg 1$. One even has $k v_{\mathrm{ti}} / \omega \ll\left(\beta_{\mathrm{i}} / 2\right)^{1 / 2}$ if the angle $\theta$ stays away from $90^{\circ}$. It is thus clear that for moderate values of $\beta_{\mathrm{i}}$ the ion thermal velocity $v_{\mathrm{ti}}$ is much smaller than the phase velocity of the whistlers. As an example, for $\beta_{\mathrm{i}}=0.22, k c / \omega_{\mathrm{pi}}=15$ and $\theta=60^{\circ}$, one obtains $k v_{\text {ti }} / \omega=5.5 \times 10^{-2}$. Hence it is appropriate to treat the ions as a cold fluid.

By contrast, the electrons' temperature has significant effects on the dispersion properties. We first examine the impact on the real part of the frequency, which is shown in Fig. 4 versus wavenumber $k$ and angle $\theta$ to the magnetic field. A contour representation is used, where the frequency expressed in units of $\Omega_{\mathrm{ci}}$ is marked on the contour. For reference, the lowest contour at $\omega / \Omega_{\mathrm{ci}}=30$ corresponds to the lower-hybrid frequency $\omega_{\mathrm{LH}}$; the one at $\omega / \Omega_{\mathrm{ci}}=300$ corresponds to the ion plasma frequency, and the one at $\omega / \Omega_{\mathrm{ci}}=450$ corresponds to half the electron cyclotron frequency, for the chosen mass ratio. The two panels compare the cold dispersion relation given by Eq. (1) (Fig. 4a) to the solution obtained from the full dispersion tensor that includes thermal effects of both ions and electrons (Fig. 4b). The agreement is rather good, except in the area shaded in light yellow. For example, if $k c / \omega_{\mathrm{pi}}=15$ and $\theta=60^{\circ}$, Eq. (1) yields $\omega=91 \Omega_{\mathrm{ci}}$. Meanwhile, the numerical solution gives $\omega=101 \Omega_{\mathrm{ci}}$ when $\beta_{\mathrm{e}}=0.22, \omega=99 \Omega_{\mathrm{ci}}$ when $\beta_{\mathrm{e}}=0.14$, and $\omega=97 \Omega_{\mathrm{ci}}$ when $\beta_{\mathrm{e}}=0.06$. Hence, there is a modest frequency increase associated with the electron pressure, yet the cold plasma Eq. (1) is fairly reliable.

What about the discrepancy in the shaded area where the wavenumbers are large? Unlike the ions, the electrons are in a kinetic regime where they can resonate with the waves and this causes damping. We have numerically solved the full dispersion relation for three electron temperatures and show the results including the damping in Fig. 5. As for Fig. 4 , contours describe the real part of the frequency $\omega / \Omega_{\mathrm{ci}}$. The associated imaginary part $\gamma$ is shown in various shades
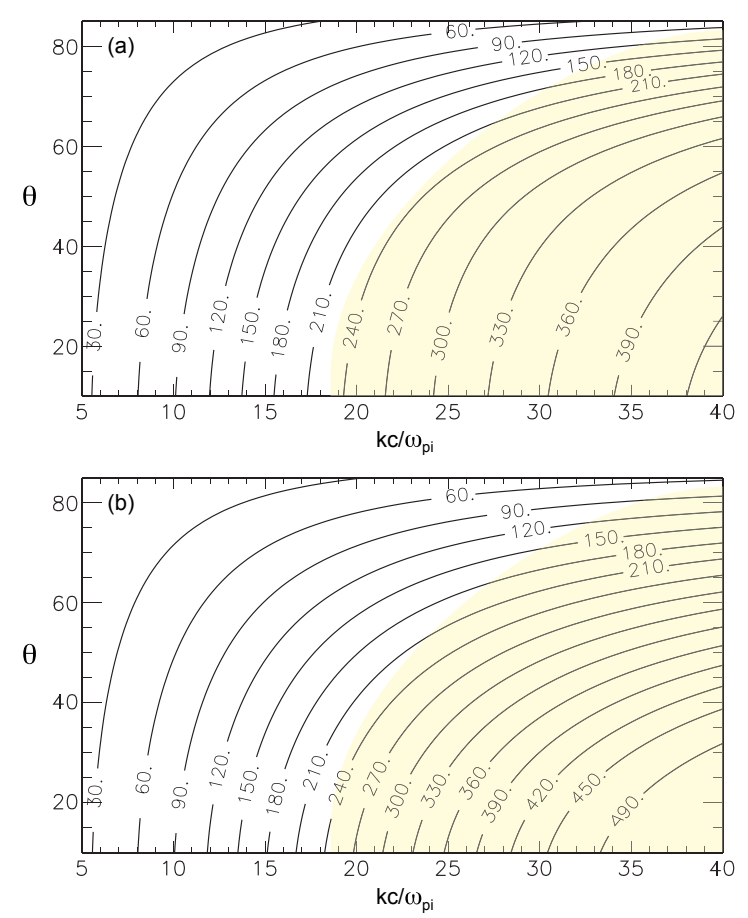

Figure 4. Solutions of the dispersion relation (without ion beam) in a linear, 2-D $[k, \theta]$ representation. Frequency is indicated by contours labeled with the value of $\omega / \Omega_{\mathrm{ci}}$. (a) Solution of the cold fluid dispersion relation given by Eq. (1); (b) real part $\operatorname{Re}(\omega)$ solution to the full kinetic dispersion tensor including thermal effects for $\beta_{\mathrm{e}}=\beta_{\mathrm{i}}=0.22$. Note the discrepancy between the two panels in the light-yellow area.

of blue. As indicated in the color code at the bottom of Fig. 5, the shades correspond to the relative damping values $\gamma / \omega$. It is evident that the damping can be very strong, in some instances resulting in quasi-modes where $|\gamma / \omega|>0.3$. Figure 5a displays the dispersion for our nominal electron temperature, $\beta_{\mathrm{e}}=0.22$. Modes in the lower-right corner are indeed heavily damped, which explains the large discrepancy in real frequencies we noted between the cold formula (Fig. 4a) and the full dispersion result (Fig. 4b).

Figure $5 \mathrm{~b}$ and $\mathrm{c}$ demonstrate how the damping progressively weakens when the electrons become colder. When $\beta_{\mathrm{e}}=0.06$ (Fig. $5 \mathrm{c}$ ) the damping drops under $5 \%$ for most wavenumbers and angles under concern. Only in the extreme lower-right corner of the plot does it exceed $5 \%$. In line with the weaker values of $\gamma / \omega$, we point out that the contours for the real frequency agree better in this instance with those of the cold formula plotted in Fig. 4a.

The terms in the full dispersion tensor are numerous and reflect different contributions. As shown in Appendix A, since the electrons are magnetized, their contribution to the tensor's elements is made of combinations of Bessel functions and derivatives with the plasma dispersion function $Z\left(\zeta_{n}\right)$, where 
(a)

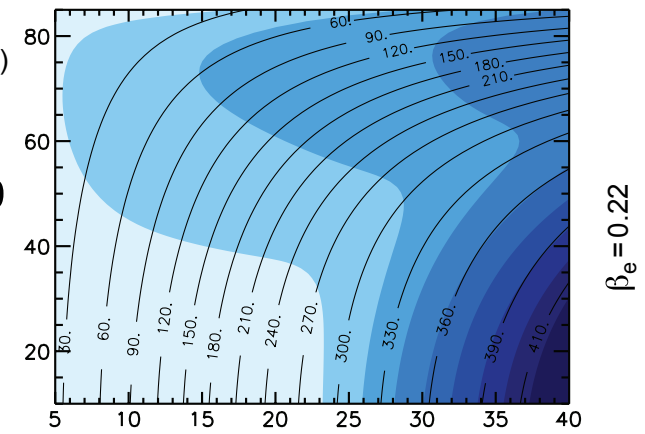

(b)
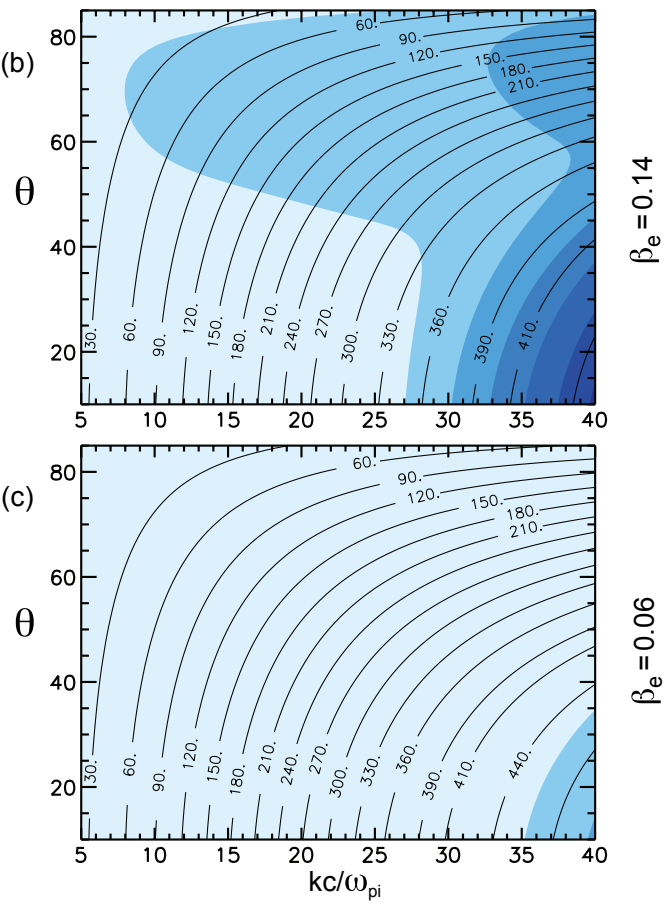

$\gamma / \omega$

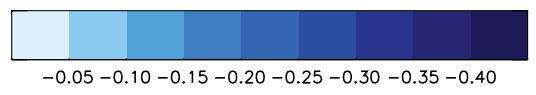

Figure 5. Solutions of the full kinetic dispersion relation (without ion beam) including thermal effects for various $\beta_{\mathrm{e}}\left(\beta_{\mathrm{i}}=0.22\right)$. Representation is similar to that of Fig. 4, with contours marking the value of $\omega / \Omega_{\text {ci }}$. In addition, the damping $\gamma$ relative to the real frequency $\omega$ is indicated by shades of blue. Note that the relative damping is substantial in this range of wavenumbers and angles except for very low $\beta_{\mathrm{e}}$.

$\zeta_{n} \equiv \frac{\omega+n \Omega_{\mathrm{ce}}}{\sqrt{2} k \cos \theta v_{\mathrm{te}}}$

We will show that the resonant velocities $v_{\text {rn }} \equiv \zeta_{n} \sqrt{2} v_{\text {te }}$ which need to be considered here are the Cerenkov velocity $v_{r 0}$ and the Doppler-shifted velocity $v_{r 1}$, respectively defined by

$v_{r 0} \equiv \frac{\omega}{k \cos \theta} \quad$ and $\quad v_{r 1} \equiv \frac{\omega-\Omega_{\mathrm{ce}}}{k \cos \theta}$.
Other resonant velocities fall outside the electron distribution function. In Fig. 6a we consider the same $[k, \theta]$ space used in previous plots, yet display here iso-contours of the Cerenkov velocity as obtained from the solution of the full dispersion relation. The values marked on the contours represent the ratio $v_{r 0} / v_{\mathrm{te}}=\sqrt{2} \zeta_{0}$, where $v_{\mathrm{te}}=\sqrt{T_{\mathrm{e}} / m}$. It is clear from the values that this resonance lies on the core of the electron distribution and so is important. What about the other resonances $(n \neq 0)$, which are shifted in velocity from $v_{r}$ by increments of $\Omega_{\mathrm{ce}} /(k \cos \theta)$ ? For the range of wavenumbers and angles considered in Fig. 5, let us evaluate the possible sizes of the increment. The smallest possible increment corresponds to modes in the lower-right corner of the plot with $k c / \omega_{\mathrm{pi}}=40$ and $\theta=10^{\circ}$. There, one has $\Omega_{\mathrm{ce}} /(k \cos \theta)=$ $2.3 v_{\text {te }}$. As one moves away toward other corners, the size of the increment grows. For example, with $k c / \omega_{\text {pi }}=40$ and $\theta=60^{\circ}$ one obtains $\Omega_{\mathrm{ce}} /(k \cos \theta)=4.5 v_{\text {te }}$, i.e., an increment already large compared to the width of the electron distribution (Fig. 6d). Thus, given that $v_{r 0}$ itself lies within the core of the distribution, typically $v_{r 0} / v_{\text {te }}<1.8$ (as illustrated in Fig. 6a), two conclusions can be drawn. First, resonances with $n \neq 0$ play a role only for modes belonging to the lower-right corner of the plot. Second, for these modes one needs to consider only the three resonances $n=-1,0,+1$. We tested that assertion by replacing in the dispersion tensor the $Z$ function by its fluid approximation (see, e.g., Swanson, 2003) for terms other than $n=-1,0,+1$. The resulting dispersion plot (not shown) looks the same as Fig. 5a. The $n=+1$ resonance interacts with electrons with a speed larger than $3 v_{\text {te }}$ and hence plays a minor role, unless the distribution includes a tail. We point out that our model of electron distribution is a simple Maxwellian. A more realistic model would include a halo. If core electrons with speed $v_{\text {te }}$ have energy of $10 \mathrm{eV}$, a halo with $100 \mathrm{eV}$ electrons would interact with the waves via the $n=+1$ resonance. We emphasize the asymmetry between $n+1$ and $n-1$ resonances. Unlike the $n+1$ resonance, the $n-1$ resonance interacts with core electrons with speeds as small as $1.4 v_{\text {te }}$. This resonance is in fact responsible for the strong damping which affects modes in the lower-right corner of Fig. 5a. Whistlers with wavevectors in the quarter disk marked with contours in Fig. 6c (i.e., wavenumbers $22<k c / \omega_{\text {pi }}<40$ and $10^{\circ}<\theta<60^{\circ}$ ) interact with the electron distribution through both $v_{r 1}$ and $v_{r 0}$. For large $k$ and small $\theta$, the $v_{r}$ resonance leads to the strong damping of the waves seen in Fig. 5a. In summary, the two important resonant velocities for damping the whistlers are the Cerenkov velocity $v_{r}$ and the Doppler-shifted velocity $v_{r 1}$.

We point out that the two resonant velocities $v_{r 0}$ and $v_{r 1}$ are in opposite directions. A given wave which has, say, a wavevector component $k_{z}=k \cos \theta>0$ is affected both by electrons moving with $v_{z}>0$ through $v_{r} 0$ and by electrons moving with $v_{z}<0$ through $v_{r 1}$. As an example, let us consider the wave with $k c / \omega_{\text {pi }}=30$ and $\theta=25^{\circ}$, whose location in Fig. $6 \mathrm{a}$ and $\mathrm{c}$ is marked with a red star. This wave interacts 

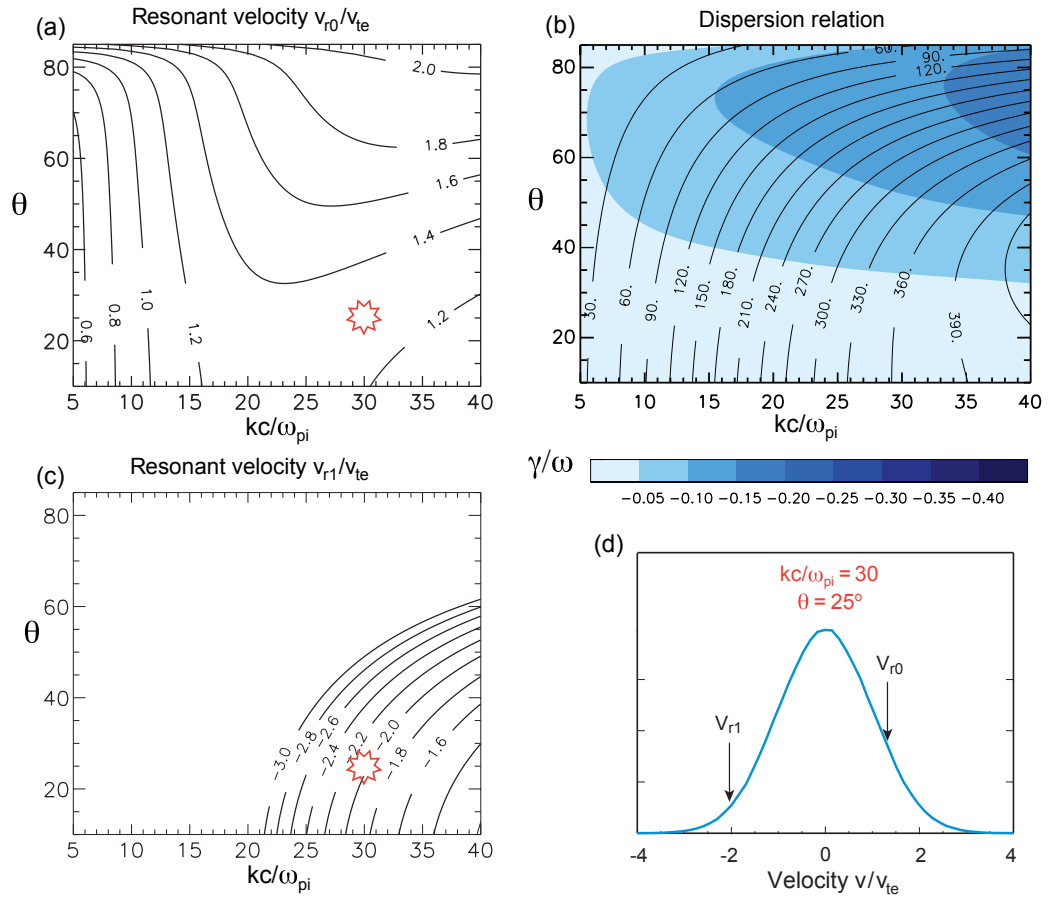

Figure 6. Composite figure illustrating the damping role of the resonant velocities on the electron distribution (for $\beta_{\mathrm{e}}=0.22$ ) in the case without ion beam. (a) Iso-contours of the Cerenkov resonant velocity $v_{r 0} / v_{\text {te }}$ (Eq. 4 ) versus $[k, \theta]$. The velocities, marked on the contours, lie on the core of the electron distribution, which indicates that the terms associated with $n=0$ in the dispersion tensor contribute to the damping (see Appendix A). (b) Solution of the dispersion relation to be compared with Fig. 5a. Here, the treatment is kinetic only for the $n=0$ terms and fluid for all others. See main text for discussion. (c) Iso-contours of the Doppler-shifted resonant velocity $v_{r} / v_{\text {te }}$ (where $v_{r 1}$ is defined for $n=-1$ in Eq. 4). (d) Location of the two resonant velocities associated with one given wave (marked with a red star in panels $\mathbf{a}$ and $\mathbf{c}$ ) in the electron distribution.

with both sides of the distribution, where many electrons can participate in its damping, as illustrated in Fig. 6d. According to quasilinear theory, we could expect that the electrons at $v_{r 0}$ are accelerated in the parallel direction, whereas the electrons at $v_{r 1}$ are accelerated in the perpendicular direction.

We will see in Sect. 3 that the two-stream instabilities occur at large angles $\left(\theta \sim 50^{\circ}\right.$ and $\left.\theta>80^{\circ}\right)$. Under these conditions the important resonance is the Cerenkov resonance $(n=0)$. In order to understand its role in damping the oblique whistlers, we replace the $Z$ function in the dispersion tensor by its fluid approximation for the $n \neq 0$ terms, keeping $Z$ for the $n=0$ terms only. The resulting dispersion relation is shown in Fig. $6 \mathrm{~b}$ (same nominal value $\beta_{\mathrm{e}}=0.22$ ), which has to be compared to Fig. 5a, where no fluid approximation is introduced. We use the same hues of blue in both plots to facilitate the comparison. Ignoring the lower-right corner for the moment, it is clear that Fig. $6 \mathrm{~b}$ reproduces the features of Fig. 5a well. One notes the same trend of increased damping with increasing wavenumber. The angular dependences of $\omega$ and $\gamma / \omega$ at fixed wavenumber are also well reproduced. From quasi-parallel to quasi-perpendicular propagation, let us stress the way the damping intensifies, reaches a maximum, and then quickly decreases as $90^{\circ}$ is approached.
Meanwhile, the resonant velocity $v_{r 0}$ remains in the bulk of the electron distribution and does not vary dramatically. What physically enters into the damping is not only the location of the resonance on the electron distribution but also the relative amplitude of the parallel component of the electric field. Larger values of $\boldsymbol{E}_{z}$ lead to more electron acceleration via the Cerenkov resonance and thus more damping of the wave. Can we evaluate the way $\boldsymbol{E}_{z}$ changes as one varies the angle $\theta$ ? In order to deal with tractable algebra, we will assume that the cold dielectric tensor is sufficient to assess this variation in polarization and judge the assumption's validity from the result obtained. The calculations which are developed in Appendix B show that indeed $\boldsymbol{E}_{z}$ varies significantly as $\theta$ increases. We repeat here just the final result, i.e., Eq. (B9):

$$
\begin{aligned}
\frac{i \boldsymbol{E}_{z}}{\boldsymbol{E}_{y}} & \approx \frac{m}{M}\left(\frac{k c}{\omega_{\mathrm{pi}}}\right)^{3} \\
& \times \frac{\left[1+\left(k c / \omega_{\mathrm{pe}}\right)^{2}\right] \cos \theta \sin \theta}{\left[1+\left(k c / \omega_{\mathrm{pe}}\right)^{2}+\left(k c / \omega_{\mathrm{pi}}\right)^{2} \cos ^{2} \theta\right]^{1 / 2}} U(k),
\end{aligned}
$$

where $U(k)$ is defined in Appendix B and is of order unity for our parameters $\left(\omega \geq \omega_{\mathrm{LH}}\right.$ and $\left.k c / \omega_{\mathrm{pi}} \geq 1\right)$. Starting from $\theta=0$ and increasing the angle, Eq. (5) shows that the par- 
allel electric field first rises, peaks, and then declines dramatically when $\theta$ approaches $90^{\circ}$. We point out that $\left|\boldsymbol{E}_{z}\right|$ maximizes at quite oblique propagation angles (e.g., $\theta \approx 70^{\circ}$ for $\left.k c / \omega_{\mathrm{pi}}=15\right)$. Furthermore, this maximum shifts closer to $90^{\circ}$ as larger wavenumbers are considered. This behavior is precisely what Fig. $6 \mathrm{~b}$ indicates for the damping associated with the Cerenkov resonance. Incidentally, the vanishing of $\boldsymbol{E}_{z}$ described by Eq. (5) in either parallel or perpendicular propagation is to be expected from the R-X mode: the polarization morphs to a circular $\mathrm{R}$ mode for $\theta \rightarrow 0^{\circ}$ and to an $\mathrm{X}$ mode for $\theta \rightarrow 90^{\circ}$. To close this section, keep in mind that the low value $\beta_{\mathrm{e}}=0.06$ assumed in Fig. 5c is unrealistic. We used it here in order to clarify the role of the electron temperature. Within the context of planetary shocks at least, our nominal value of $\beta_{\mathrm{e}}=0.22$ is more probable. Thus, oblique whistlers are indeed damped by the electrons and, if an instability occurs, this damping has to be overcome.

\section{Unstable oblique whistlers due to a drifting ion population}

Consider now the situation where an ion population is drifting at speed $V_{\mathrm{d}}$ with respect to the electrons, as depicted in Fig. 1 for the core and the reflected beam. In the following, $V_{\mathrm{d}}$ can represent either the relative fast drift of the reflected ions/electrons or the relative slow drift of the core/electrons. A simple way to understand the possible instabilities that can result is to return to the cold plasma model.

\subsection{Cold plasma and cold beam}

We revert to Fig. 3 for the three angles considered $(\theta=10$, $55,85^{\circ}$ ), on which we superpose the Doppler frequency associated with a drift $V_{\mathrm{d}}$,

$\omega=k \sin \theta V_{\mathrm{d}}$.

The result is shown in Fig. 7a for a drift $V_{\mathrm{d}}=8 V_{\mathrm{A}}$. The same color convention used in Fig. 3 distinguishes the various propagation angles: $\theta=10^{\circ}$ (green), $55^{\circ}$ (blue), and $85^{\circ}$ (red). Couplings between the beam mode and the oblique whistlers, and hence possible instabilities, can only occur at locations where the triplet $(\omega, k, \theta)$ is the same. Thus, we look for the intersections of dashed curves (beam mode) with solid curves (whistler) of the same color. For quasi-parallel waves $\left(\theta=10^{\circ}\right.$ in green), there is no intersection because the beam mode is at a frequency lower than the whistler everywhere. Conversely, for quasi-perpendicular waves $\left(\theta=85^{\circ}\right.$ in red), there is no intersection because the beam mode is at a frequency higher than the whistler everywhere. Nonetheless, a coupling to another wave mode is possible for such a large angle. The first Bernstein branch, which is indicated here in purple, intersects the beam mode at a high wavenumber $k \rho_{\mathrm{e}}>1$. The location is marked ECDI for the electron cyclotron drift instability. In Sect. 4 we will discuss the question
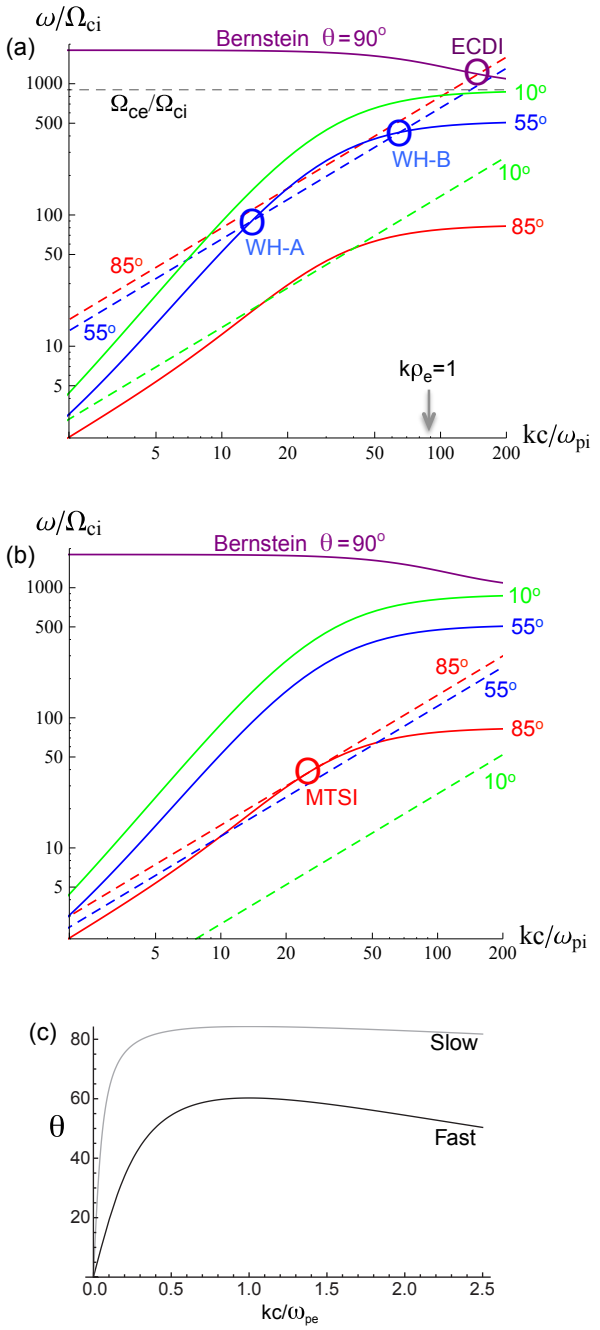

Figure 7. Similar to Fig. 3 with the addition of beam modes (colored dashed lines) described by Eq. (6) for the three angles considered: $\theta=10,55,85^{\circ}$. (a) Example of a fast drift $V_{\mathrm{d}}=8 V_{\mathrm{A}}$. Possible couplings of the beam modes to the oblique whistlers (same triplet $(\omega, k, \theta))$ can only occur at locations marked WH-A and WH-B. Coupling to the Bernstein wave is at ECDI; see text for details. (b) Example of a slow drift $V_{\mathrm{d}}=1.5 V_{\mathrm{A}}$. As compared to (a), the frequency of the beam modes is now lowered. As a result, possible couplings to the oblique whistlers can only occur for very oblique propagation at the location marked MTSI; see text for details. (c) Plot of Eq. (7), where $\kappa_{\mathrm{e}} \equiv k c / \omega_{\text {pe }}$ for the two values of $V_{\mathrm{d}}=1.5 V_{\mathrm{A}}$ and $8 V_{\mathrm{A}}$.

of this instability for angles $\theta$ that are off $90^{\circ}$. At this point, we just note how much larger is the frequency of the ECDI than any frequency of a whistler propagating at $\theta=85^{\circ}$ (full red curve).

When whistlers propagate obliquely (for example here $\theta=55^{\circ}$ in blue) two intersections exist because their dispersion relation $\omega(k)$ displays some curvature. The intersections are marked as WH-A and WH-B in Fig. 7a. Following Eq. (6), their locations depend upon $V_{\mathrm{d}}$. WH-A and WH-B 
come closer to each other as $V_{\mathrm{d}}$ increases and the dashed line moves up. Varying $\theta$ leads to a similar, albeit a little more complex, change since both Eqs. (6) and (1) depend upon $\theta$.

We now derive an equation that describes the locations of all WH intersections in a $[k, \theta]$ space. One defines the wavenumber in terms of electron inertia length, $\kappa_{\mathrm{e}}=k c / \omega_{\text {pe }}$, and introduces the parameter $\alpha \equiv\left(M_{\mathrm{i}} / m_{\mathrm{e}}\right)^{1 / 2}\left(V_{\mathrm{A}} / V_{\mathrm{d}}\right)$ for the drift. Assuming $\left(\cos \theta k c / \omega_{\mathrm{pi}}\right)^{2} \gg 1$, one can combine Eqs. (6) and (1) to yield the explicit relation

$\theta\left(\kappa_{\mathrm{e}}\right)=\arctan \left(\frac{\alpha \kappa_{\mathrm{e}}}{1+\kappa_{\mathrm{e}}^{2}}\right)$.

The profile $\theta\left(\kappa_{\mathrm{e}}\right)$ is shown in Fig. 7c for two values of the parameter $\alpha$. Where $\kappa_{\mathrm{e}}<1$ it rises at a rate controlled by $\alpha$ : the slower the drift, the steeper the slope. The maximum $\theta^{*}$ is reached for $\kappa_{\mathrm{e}}=1$, beyond which the profile slowly decreases. The value of $\theta^{*}$ is given by

$\theta^{*}=\arctan \left(\frac{\alpha}{2}\right)=\arctan \left[\left(\frac{M_{\mathrm{i}}}{4 m_{\mathrm{e}}}\right)^{1 / 2} \frac{V_{\mathrm{A}}}{V_{\mathrm{d}}}\right]$.

Hence, for a given propagation angle $\theta<\theta^{*}$, Eq. (7) can be inverted to yield two possible wavenumbers:

$\kappa_{\mathrm{e}}=\frac{\alpha}{2} \cot \theta \pm\left(\frac{\alpha^{2}}{4} \cot ^{2} \theta-1\right)^{1 / 2}$.

These lower and higher wavenumbers correspond to the locations marked WH-A and WH-B, respectively, in Fig. 7a. When the angle reaches $\theta=\theta^{*}$, they merge at the location defined by $\kappa_{\mathrm{e}}=k c / \omega_{\mathrm{pe}}=1$ and $\omega=\omega_{\mathrm{LH}}\left(V_{\mathrm{A}}^{2} / V_{\mathrm{d}}^{2}+\right.$ $\left.4 m_{\mathrm{e}} / M_{\mathrm{i}}\right)^{-1 / 2} \approx \omega_{\mathrm{LH}}\left(V_{\mathrm{d}} / V_{\mathrm{A}}\right)$, where $\omega_{\mathrm{LH}} \equiv\left(\Omega_{\mathrm{ci}} \Omega_{\mathrm{ce}}\right)^{1 / 2}$. For angles larger than $\theta^{*}$, no intersection exists; hence, no instability is possible with the given drift. For example, in the case shown in Fig. 7a, where the drift is $V_{\mathrm{d}}=8 V_{\mathrm{A}}$, the maximum angle is $\theta^{*}=62^{\circ}$.

Let us now suppose that we want to destabilize waves propagating at very large angles, close to $\theta \approx 90^{\circ}$. Equation (8) can be inverted to find the required drift. The latter clearly needs to be modest, namely $V_{\mathrm{d}} \gtrsim V_{\mathrm{A}}$, a condition that can be satisfied by the ion core drifting versus the electrons (see Fig. 1). The case is shown in Fig. 7b, which is drawn for $V_{\mathrm{d}}=1.5 V_{\mathrm{A}}$. No intersection exists at either $\theta=10^{\circ}$ (green) or $\theta=55^{\circ}$ (blue). On the other hand, at $\theta=85^{\circ}$ the two roots on the red curve are indeed merging, the wavenumber is close to $k c / \omega_{\text {pe }}=1$ (or $k c / \omega_{\text {pi }}=30$ for the chosen mass ratio $\left.M_{\mathrm{i}} / m_{\mathrm{e}}=900\right)$ and the frequency is close to $\omega=1.5 \omega_{\mathrm{LH}}$. This instability which has a propagation angle close to $90^{\circ}$ is known in the literature as MTSI which stands for modified two-stream instability (e.g., McBride et al., 1972; Matsukiyo and Scholer, 2003; Umeda et al., 2012). Hence, we have marked it correspondingly in Fig. 7. Because the waves are destabilized by the drift of the ion core versus the electrons, these waves clearly propagate in a direction opposite to the oblique whistlers of WH-A and the Bernstein waves of the ECDI. As for the other whistlers at location WH-B, we will show in Sect. 3.2 that they are strongly damped by the electrons once thermal effects are taken into account.

\subsection{Warm plasma and warm beam}

Section 2.2 has stressed that a population of warm electrons substantially damps the obliquely propagating whistlers at most wavenumbers and angles. The damping rate even becomes very strong for large wavenumbers (see Fig. 5a). In order to have an effective instability, this damping rate needs to be overcome by a sufficient growth rate driven by the ion drift. We investigate the question by numerically solving the full electromagnetic dispersion relation for varying parameters. In the spirit of the lower-hybrid regime, the ions are taken as unmagnetized, whereas the electrons are magnetized. We work in the frame where the total momentum density vanishes, sometimes called the "proper frame". We emphasize that the drift and density values of the reflected ion beam and the incoming ion core cannot be arbitrarily chosen yet must satisfy the zero current condition for an application to the shock's foot, namely $n_{\mathrm{b}} V_{\mathrm{b}}+n_{\mathrm{c}} V_{\mathrm{c}}=0$. Henceforth, the values selected for the numerical results that follow differ slightly from those discussed in Sect. 3.1. The parameter values, which are summarized in Table 2, assume that the reflected ion beam of Fig. 1 has density $n_{\mathrm{b}}=0.2 n_{\mathrm{e}}$ and drifts at speed $V_{\mathrm{b}}=10 V_{\mathrm{A}}$ versus the electron population at rest. The incoming ion core drifts in the opposite direction with $V_{\mathrm{c}}=-2.5 V_{\mathrm{A}}$ and has a density $n_{\mathrm{c}}=0.8 n_{\mathrm{e}}$. Both ion populations have the same thermal velocity $v_{\mathrm{tc}}=v_{\mathrm{tb}}$ with $\beta_{\mathrm{c}}+\beta_{\mathrm{b}}=0.22$. Electron temperature will vary as specified. Details concerning the dielectric tensor entering our calculations can be found in Appendix A.

\subsubsection{Reflected ion beam}

Let us first examine the case where an ion population drifts fast with respect to the electrons, a situation represented by the reflected beam in Fig. 1b. The cold model then predicts two zones of instability for oblique whistlers at fixed $\theta$ which are labeled WH-A and WH-B in Fig. 7a. Now, from the study on electron damping and Fig. 5a, we can expect that whistlers in WH-B are more damped than those in WH-A due to their larger wavenumbers. That is indeed the case, as illustrated in Fig. 8, which shows the effect of the electron temperature. Profiles of $\gamma(k)$ at the fixed propagation angle $\theta=50^{\circ}$ are displayed for two $\beta_{\mathrm{e}}$ values. When electrons are very cold, namely $\beta_{\mathrm{e}}=0.02$, the profile exhibits the two zones of growth $(\gamma>0)$ corresponding to WH-A and WH-B, as expected from the cold model. However, the electron temperature has a dramatic effect, since already with $\beta_{\mathrm{e}}=0.04$ the instability at high wavenumbers (for $k c / \omega_{\text {pi }}>40$ ) is suppressed due to electron damping $(\gamma<0)$.

Numerical solutions of the dispersion relation in $[k, \theta]$ space for two electron temperatures $\left(\beta_{\mathrm{e}}=0.06,0.14\right)$ are dis- 
Table 2. Species characteristics in normalized units.

\begin{tabular}{llll}
\hline & Electrons & Ion core & Ion beam \\
\hline density & $n_{\mathrm{e}}=1.0$ & $n_{\mathrm{c}}=0.8$ & $n_{\mathrm{b}}=0.2$ \\
Drift & $V_{\mathrm{e}}=0$. & $V_{\mathrm{c}}=-2.5$ & $V_{\mathrm{b}}=+10$ \\
Gyrofrequency & $\Omega_{\mathrm{ce}}=900$ & $\Omega_{\mathrm{cc}}=1$ & $\Omega_{\mathrm{cb}}=1$ \\
Plasma frequency & $\omega_{\mathrm{pe}}=9000$ & $\omega_{\mathrm{pc}}=268$ & $\omega_{\mathrm{pb}}=134$ \\
Gyroradius & $\rho_{\mathrm{e}}=0.0111$ & $\rho_{\mathrm{c}}=0.332$ & $\rho_{\mathrm{b}}=0.332$ \\
Debye length & $\lambda_{\mathrm{de}}=1.22 \times 10^{-3}$ & $\lambda_{\mathrm{dc}}=1.24 \times 10^{-3}$ & $\lambda_{\mathrm{db}}=2.47 \times 10^{-3}$ \\
Temperature & $T_{\mathrm{e}}=1$ & $T_{\mathrm{c}}=1$ & $T_{\mathrm{b}}=1$ \\
Thermal velocity & $v_{\mathrm{te}}=10$ & $v_{\mathrm{tc}}=0.332$ & $v_{\mathrm{tb}}=0.332$ \\
Beta & $\beta_{\mathrm{e}}=0.22$ & $\beta_{\mathrm{c}}=0.197$ & $\beta_{\mathrm{b}}=0.0984$ \\
\hline
\end{tabular}

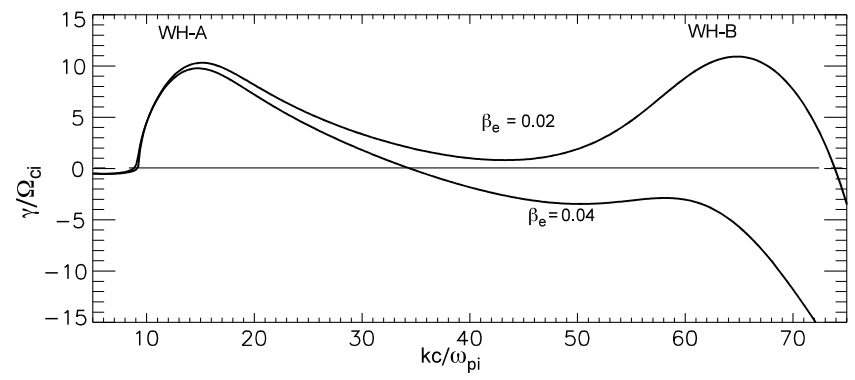

Figure 8. Effect of the electron temperature on the growth rate. Solution of the full dispersion relation for oblique whistlers $\left(\theta=50^{\circ}\right.$ fixed) destabilized by an ion beam drifting at $V_{\mathrm{d}}=10 V_{\mathrm{A}}$ (parameters of Table 2). Of the two potential candidates for growth, WH-A and WH-B (see Fig. 7a), the instability at high wavenumbers is dramatically quenched $(\gamma<0)$ by even slightly warm electrons (compare $\beta_{\mathrm{e}}=0.04$ with $\beta_{\mathrm{e}}=0.02$ ).

played in Fig. 9a, b. We use red hues to indicate growth rates and blue hues for damping rates. In addition, solid contours as in previous plots mark the real frequency $\omega / \Omega_{\text {ci }}$. Figure $9 \mathrm{a}$ and $\mathrm{b}$ exhibit unstable and damped domains and demonstrate that the electron temperature quenches the unstable locations at high wavenumbers. Recall Fig. 7c of the cold model and its predicted profile for the unstable locations in the $[k, \theta]$ space. This profile is noticeable through the reddish area in Fig. 9a and has a maximum at about $\theta \sim 59^{\circ}$ and $k c / \omega_{\text {pi }} \sim 30$, viz. $k c / \omega_{\text {pe }} \sim 1$, as predicted by Eq. (7). The plot is drawn for the same very cold temperature $\beta_{\mathrm{e}}=0.06$ used in Fig. 5c. When electrons warm up, more of the high wavenumbers are stabilized as demonstrated with Fig. 9b. The maximum growth rate is then displaced toward lower wavenumbers: here $k c / \omega_{\text {pi }} \sim 20$ and $\theta \sim 55^{\circ}$ for $\beta_{\mathrm{e}}=0.14$. For even warmer electrons such as for our nominal electron temperature of $\beta_{\mathrm{e}}=0.22$, the unstable domain is further shifted toward lower wavenumbers with almost no instability beyond $k c / \omega_{\text {pi }} \sim 30$, viz. $k c / \omega_{\text {pe }} \sim 1$. Figure 9c provides a zoomed-in view of the unstable domain in this instance. The most unstable waves (dark-red area) have $k c / \omega_{\mathrm{pi}}=16.5 \pm 4.5$ and $\theta=53^{\circ} \pm 4^{\circ}$.

\subsubsection{Incoming ion core}

Section 3.1 has demonstrated that the relatively slow drift of the incoming ion core/electrons enables destabilization of quasi-perpendicularly propagating whistlers with frequencies around $\omega_{\mathrm{LH}}$. We have numerically solved the warm dispersion relation in the case where the ion core with a relative density $n_{\mathrm{c}}=0.8 n_{\mathrm{e}}$ drifts at $V_{\mathrm{d}}=2.5 V_{\mathrm{A}}$. Figure 10 provides a zoomed-in view of the domain of maximum growth in $[k, \theta]$ space. As previously, red hues indicate growth rates and blue hues damping rates. Comparing this plot to Fig. 9c (fast drift case), one notes two points: first, the growth rate is somewhat larger; second, the aspect ratio of the plot is quite different. The wavenumber range is more extended yet the angular range is more limited. This feature is in fact consistent with the profile $\theta\left(\kappa_{\mathrm{e}}\right)$ of Fig. 7c. The maximum growth is now centered about wavenumbers $k c / \omega_{\text {pi }}=30$ (or $\kappa_{\mathrm{e}} \equiv$ $\left.k c / \omega_{\text {pe }}=1\right)$, where the profile is quite flat. Hence, the corresponding spread $\delta\left(k c / \omega_{\text {pi }}\right)$ is large, whereas the spread $\delta \theta$ is limited to a few degrees. By contrast, in the fast drift case of Fig. 9c the maximum growth is centered about wavenumbers $k c / \omega_{\mathrm{pi}}=16.5$ (or $\kappa_{\mathrm{e}} \equiv k c / \omega_{\mathrm{pe}}=0.55$ ). Here the profile marked "fast" has a clear positive slope, which leads to a larger $\delta \theta$ spread. Accordingly, the numerical solution plotted in Fig. 9c shows the angular spread to be at least $10^{\circ}$.

\section{Perpendicular/quasi-perpendicular electromagnetic ECDI}

Reverting to the schematic plot in Fig. 7a, which treats the case where an ion population drifts fast with respect to the electrons, one sees that for nearly perpendicular propagation (here $\theta=85^{\circ}$ in red) the beam mode can intersect the first Bernstein branch. The frequency is near $\Omega_{\text {ce }}$ and the wavenumber near $k \rho_{\mathrm{e}} \sim v_{\mathrm{te}} /\left(V_{\mathrm{d}} \sin \theta\right) \sim 1$. Of course, Bernstein modes are commonly defined with wavevectors at exactly $90^{\circ}$ to $\boldsymbol{B}_{o}$ and experience no damping. However, they belong to a larger class of modes, called the electron cyclotron harmonic waves (ECHWs), which can propagate in a narrow angular range between $\theta_{o}$ and $90^{\circ}$ with minimal 

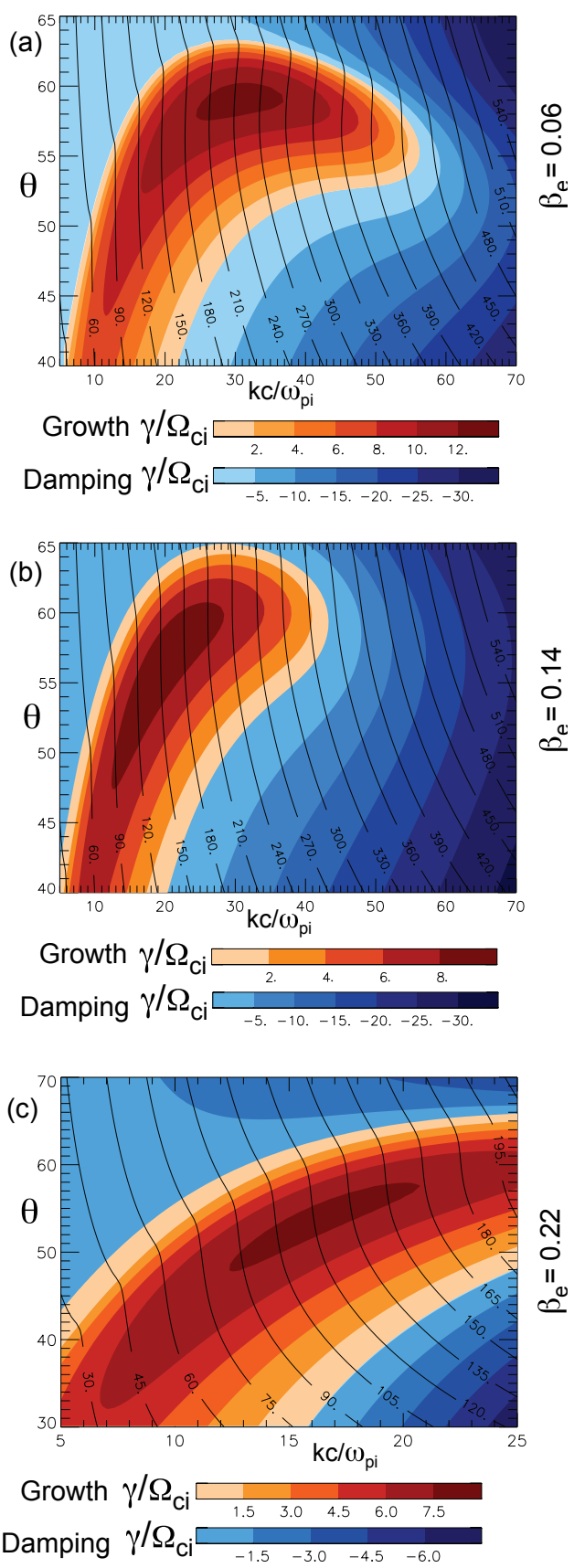

Figure 9. Numerical solutions of the dispersion relation in $[k, \theta]$ space for the case of a fast ion beam with $V_{\mathrm{d}}=10 V_{\mathrm{A}}$ and $n_{\mathrm{b}} / n_{\mathrm{e}}=$ 0.2 . Zones made unstable by the beam are marked with red hues, while blue hues denote damping. Note the changes in the color code between the three panels $(\mathbf{a}, \mathbf{b}, \mathbf{c})$. The real frequency is indicated by contours labeled with the value of $\omega / \Omega_{\text {ci }}$. An increased electron temperature quenches the instability at the higher wavenumbers (compare panels a and b). (c) Zoomed-in view of the unstable region for $\beta_{\mathrm{e}}=0.22$, relevant temperature for the simulation presented in Sect. 5.2.

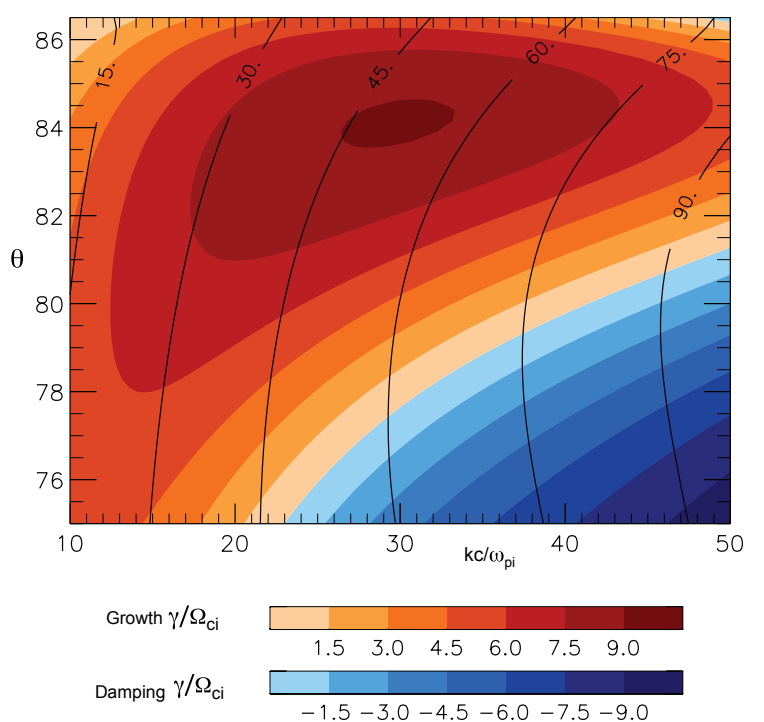

Figure 10. Zoomed-in view similar to Fig. 9c for a slow drift case such as the ion core with $V_{\mathrm{d}}=2.5 V_{\mathrm{A}}, n_{\mathrm{c}} / n_{\mathrm{e}}=0.8$, and $\beta_{\mathrm{e}}=0.22$.

damping as compared to their frequencies (Lembege, 1979a, and references therein). The angle $\theta_{o}$ is typically a few degrees less than $90^{\circ}$ and depends upon the wavenumber and the frequency. For $\theta<\theta_{o}$ the damping increases dramatically due to the Doppler-shifted resonant velocity on the electrons. Incidentally, the direction of the ECHW's group speed is given by the normal to the undamped part of the $\left(k_{\perp}, k_{\|}\right)$diagram. This undamped part can be either convex or concave depending on the wave frequency ratio $\omega / \Omega_{\mathrm{ce}}$ within a given dispersion branch, which allows the direction of the group speed to cover a very large spatial range. This explains why the waves can be spatially detected in all directions in real space around a source, even though low-damped wavevectors are themselves confined within the narrow angular range $\left(\theta_{o}, 90^{\circ}\right)$ in $k$ space (Lembege and Gonfalone, 1978; Lembege, 1979b; Thiel and Lembège, 1982).

For the present work, the important feature to note is the quasi-absence of damping for waves with $\theta>\theta_{o}$, which means that the waves can exist in a cone angle about $90^{\circ}$ that is finite and not zero as often believed. This is illustrated in Fig. 11a, which displays dispersion results computed numerically with the full EM tensor (see Appendix A) for directions off perpendicular. One sees that the damping is less than $10^{-3} \Omega_{\mathrm{ce}}$ (lighter hue of blue) for a few degrees off $\theta=90^{\circ}$. In the presence of an ion beam, this damping can easily be overcome, leading to unstable modes. Figure $11 \mathrm{~b}$ shows the area in $[k, \theta]$ space that is unstable due to the same ion beam we used in Fig. 9 for the oblique whistlers. Beam parameters are $n_{\mathrm{b}} / n_{\mathrm{e}}=0.2$ and $V_{\mathrm{d}} / V_{\mathrm{A}}=$ 10. The maximum growth rates (marked with dark-red hues) are $\gamma>3 \times 10^{-2} \Omega_{\mathrm{ce}}$. These are larger than the rates obtained for the oblique whistlers, which peak at $\gamma \approx 8 \Omega_{\mathrm{ci}}$, by over a factor of 3 . 

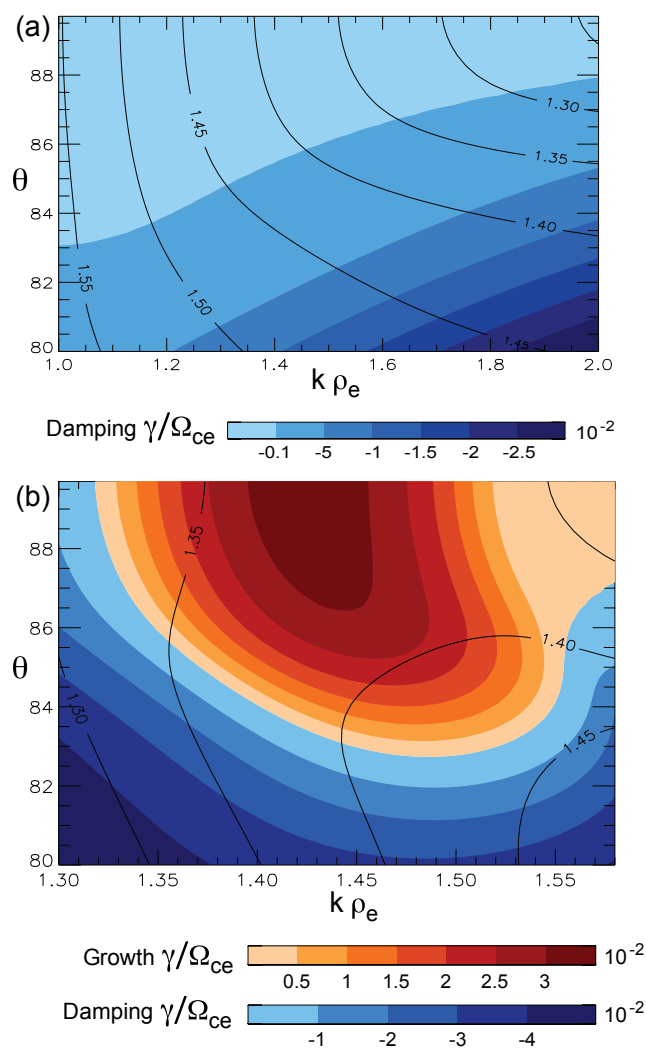

Figure 11. Waves with much larger wavenumbers and frequencies than those in Fig. 9c can also be destabilized by the same ion beam (with $V_{\mathrm{d}}=10 V_{\mathrm{A}}$ and $n_{\mathrm{c}} / n_{\mathrm{e}}=0.2$ ). They belong to an extension of the first Bernstein branch and are not strictly perpendicular. Dispersion relation without (a) and with (b) the ion beam. Real frequencies are indicated by contours labeled with the values of $\omega / \Omega_{\mathrm{ce}}$. Wavenumbers are normalized by the electron gyroradius $\rho_{\mathrm{e}}$. Damping (due to electrons) is indicated by hues of blue, while the zones made unstable by the beam are marked with red hues.

\section{Simulations}

\subsection{Description of 1-D PIC simulation}

We employ a 1-D electromagnetic PIC code with periodic boundary conditions and initially load a plasma made of the three components depicted in Fig. 1b. The simulation is carried out in the same frame as used for the dispersion analysis, often known as the proper frame, where the total momentum vanishes. The components, namely electrons, ion beam, and ion core, are given identical characteristics (defined in Table 2) to those used for the dispersion computations of Sects. 3.2 and 4. In the spirit of lower-hybrid instabilities, the simulation treats the ions as unmagnetized, whereas the electrons are magnetized. Since we are restricted to a 1-D $3 \mathrm{~V}$ code, the direction of the unstable wavevector $\boldsymbol{k}$ is predetermined by the simulation setup to one spatial direction (1D), while particle velocities can have three spatial directions $(3 \mathrm{~V})$. Oblique simulations are performed where the angle $\theta$ between $\boldsymbol{k}$ and the background magnetic field $\boldsymbol{B}_{O}$ is predefined following the information obtained from the dispersion analysis in Sects. 3-4. Figure 2 shows in blue the orientation of the three axes associated with the simulations. With respect to the system of axes defined by the direction of $\boldsymbol{V}_{\mathrm{d}}$ and $\boldsymbol{B}_{o}$ used in previous sections, the simulation system is rotated by $\pi / 2-\theta$ within the plane $\left[\boldsymbol{k}, \boldsymbol{B}_{o}\right]$ and composed of the directions $L$ (for longitudinal field) along the wave vector and two directions $t_{1}$ and $t_{2}$ (for transverse field). The direction $t_{2}$ is contained in the plane $\left[\boldsymbol{k}, \boldsymbol{B}_{o}\right]$ and thus partially projects along $\boldsymbol{B}_{o}$, while $t_{1}$ is perpendicular to the plane and has no projection along $\boldsymbol{B}_{o}$.

Two series of simulations are performed - one along an oblique direction $\theta=55^{\circ}$ and one along a quasiperpendicular direction $\theta=84^{\circ}$. The angle $\theta=55^{\circ}$ is chosen to capture the oblique whistlers destabilized by the fast ion beam which have maximum growth according to Fig. 9c. The angle $\theta=84^{\circ}$ is chosen to capture the quasiperpendicular whistlers destabilized by the slow ion core which have the maximum growth according to Fig. 10. The box is long enough to include the most unstable wavelengths for the oblique whistlers as predicted from Fig. 9c. The grid consists of 8192 cells and spans $16384 \lambda_{\text {de }}$, where $\lambda_{\text {de }}$ is the electron Debye length (or equivalently 546 electron inertia lengths or 18 ion inertia lengths). The grid cells are small enough (cell size $\Delta=2 \lambda_{\mathrm{de}}$ ) to describe the short-wavelength Bernstein waves expected from Fig. $11 \mathrm{~b}\left(k \rho_{\mathrm{e}}=1.4\right.$ implies a wavelength $\lambda \approx 45 \lambda_{\mathrm{de}}$ ). The electric and magnetic fields have five components: $E_{t_{1}}, E_{t_{2}}, E_{\mathrm{L}}, B_{t_{1}}$, and $B_{t_{2}}$, where $E_{\mathrm{L}}$ is the longitudinal component satisfying Poisson equation. Finally, we use 2000 macroparticles per population (ion beam, ion core, and electrons) and per cell for achieving good statistics.

The simulation results presented here are preliminary due to the use of a 1-D code. Our objectives are twofold. First, we want to confirm by actual PIC results the dispersion analysis which makes up the bulk of this paper. Second, the aim is to "calibrate" the various instabilities and prepare the stage for 2-D simulations to come. In this light the simulation runs will be limited to the "linear" stage. The nonlinear stage has to be addressed with 2-D simulations since coupling between waves propagating at various angles can be expected in this regime.

\subsection{Unstable oblique whistlers}

Here, we set $\theta=55^{\circ}$ and run the simulation for several hundreds of $\Omega_{\mathrm{ce}}^{-1}$, or a few tens of $\omega_{\mathrm{LH}}^{-1}$, while the instability is in its "linear" regime. A sample of the results is visible in Figs. 12 and 13. The wave energy is heavily dominated by the magnetic part. We normalized the latter to the kinetic energy of the electrons at $t=0$ and show its time history in Fig. 12a. A power spectrum is shown at time $t_{\mathrm{A}}=780 \Omega_{\mathrm{ce}}^{-1}$ in Fig. 12b. The power is clearly concentrated in wavenumbers around $k c / \omega_{\mathrm{pi}}=18$, as expected from the dispersion 


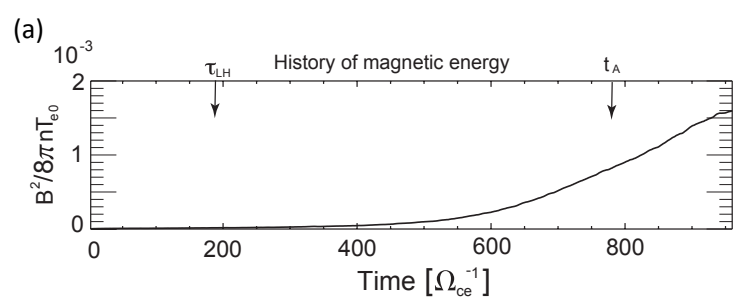

(b)
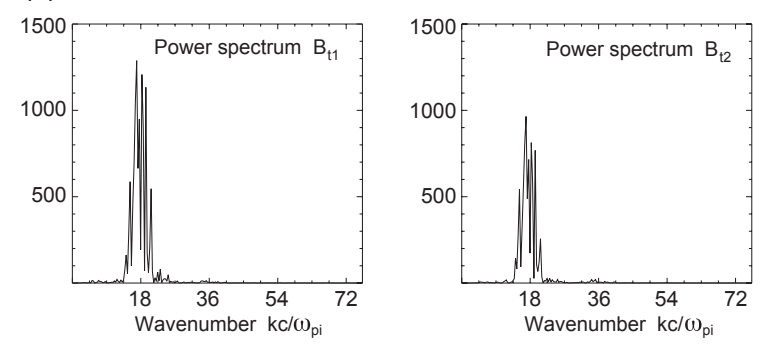

Figure 12. Whistler instability for oblique propagation, $\theta=55^{\circ}$ and $\beta_{\mathrm{e}}=0.22$. (a) Time history of the magnetic wave energy, which shows the slow development of the instability. For reference, we show the time $\tau_{\mathrm{LH}} \equiv 2 \pi / \omega_{\mathrm{LH}}$, which was characteristic of our previous simulations on the ECDI (Muschietti and Lembège, 2013). Note that the ion cyclotron period $\tau_{\mathrm{ci}}=5.7 \times 10^{3} \Omega_{\mathrm{ce}}^{-1}$ is much longer than the present simulation's time. (b) Power spectra of the two magnetic components $B_{t_{1}}$ and $B_{t_{2}}$ at time $t_{\mathrm{A}}=780 \Omega_{\mathrm{ce}}^{-1}$ indicated in (a). The spectra agree with the results obtained from the dispersion study: wavenumbers extend over the range $14<k c / \omega_{\text {pi }}<$ 22 (destabilized by the fast drift of the ion beam).

analysis shown in Fig. 9c. One can also observe that the magnetic component $B_{t_{1}}$ is somewhat larger than $B_{t_{2}}$. This feature is consistent with the asymmetry noted above between the two transverse directions. For wave frequencies in the lower-hybrid range, the electrons are magnetized, whereby their motion in the direction $t_{1}$ is inhibited as compared to that in the direction $t_{2}$, which has a component along $\boldsymbol{B}_{o}$. One can therefore expect the electron current $j_{t_{1}}$ to be smaller than $j_{t_{2}}$, resulting in $B_{t_{1}}$ being larger than $B_{t_{2}}$.

Figure 13 further documents the characteristics of the waves excited in the simulation. In order to check whether the frequencies are those expected from the dispersion analysis, we set up probes at fixed positions and register the local magnetic field versus time. A sample of such a record is shown in Fig. 13a, which exhibits oscillations with a period on the order of $40 \Omega_{\mathrm{ce}}^{-1}$. The associated power spectrum is shown in Fig. 13b, where the frequency is normalized with respect to $\Omega_{\mathrm{ci}}$ for an easy comparison with the frequencies of Fig. 9c. The power is peaked at $\omega \approx 140 \pm 10 \Omega_{\mathrm{ci}}$, which is in very good agreement with the dispersion study that predicts a frequency $\omega=138 \Omega_{\mathrm{ci}}$ for $k c / \omega_{\mathrm{pi}}=18$ and $\theta=55^{\circ}$.

Another feature to analyze is the polarization of the excited waves. To this end, we construct hodograms of the magnetic and transverse electric fields by means of the following procedure. The components $B_{t_{1}}, B_{t_{2}}, E_{t_{1}}$, and $E_{t_{2}}$ are assumed to have a phase which varies according to
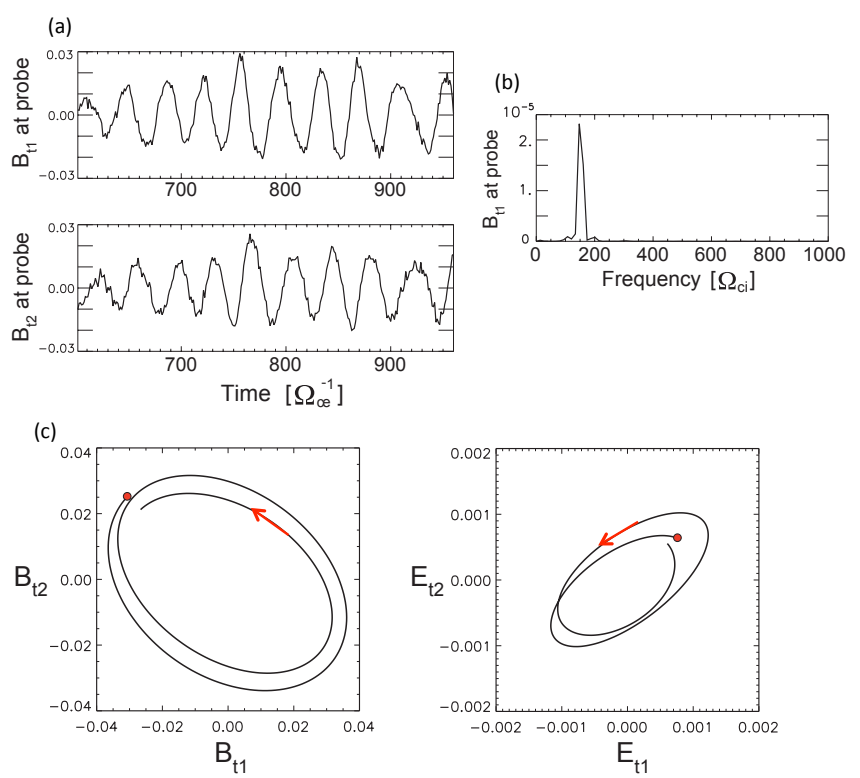

Figure 13. Whistler instability for oblique propagation, $\theta=55^{\circ}$ and $\beta_{\mathrm{e}}=0.22$. (a) Time variation of the wave components $B_{t_{1}}$ and $B_{t_{2}}$. (b) Power spectrum showing the frequency $\omega \sim 140 \pm$ $10 \Omega_{\mathrm{ci}}$, in agreement with predictions from the dispersion analysis. (c) Hodograms of the transverse electric and magnetic fields after filtering (see text). The direction of rotation follows the electron gyromotion, which confirms the whistler nature of the wave.

$(k \xi-\omega t+\phi)$, where $\xi$ measures the distance along the wave normal and $\phi$ is a constant specific to each component. After examining snapshots at increasing times we safely conclude that the phase speed is positive; hence, $k$ and $\omega$ are positive numbers. Now, one can construct hodograms either by fixing $\xi$ and letting the time $t$ vary or, conversely, by fixing $t$ and letting $\xi$ vary. We choose the latter method because of a better data sampling. The magnetic signal in $\xi$ evidences very clear waveforms which are similar to those shown in Fig. 13a and translates in the well-peaked power spectra shown in Fig. 12b. On the other hand, the electric signal is very noisy even with the large number of macroparticles employed. Its power spectrum (not shown here) nonetheless includes a peak at $k c / \omega_{\mathrm{pi}}=18$, which is clearly associated with that in the magnetic spectrum. Therefore, in order to deal with the noisy electric signal, we design a filter centered around $k c / \omega_{\mathrm{pi}}=18$ that is wide enough to fit the magnetic spectrum and its well-defined peak. The filter is then applied to the electric signal in order to isolate the electric part, which is related to the magnetic waveforms. Figure $13 \mathrm{c}$ displays the resulting hodograms. The direction of the field rotation, indicated by a red arrow, corresponds to that of the electron gyromotion. Therefore the wave is right-polarized, which confirms its whistler nature. Applying Faraday's law enables us to check another point for consistency. Since $B_{t} / E_{t}=c / v_{\mathrm{ph}}$, we can independently estimate the phase speed $v_{\mathrm{ph}}$ of the waves. One obtains $v_{\mathrm{ph}}=c / 35$, which yields $v_{\mathrm{ph}}=8.6 V_{\mathrm{A}}$ 

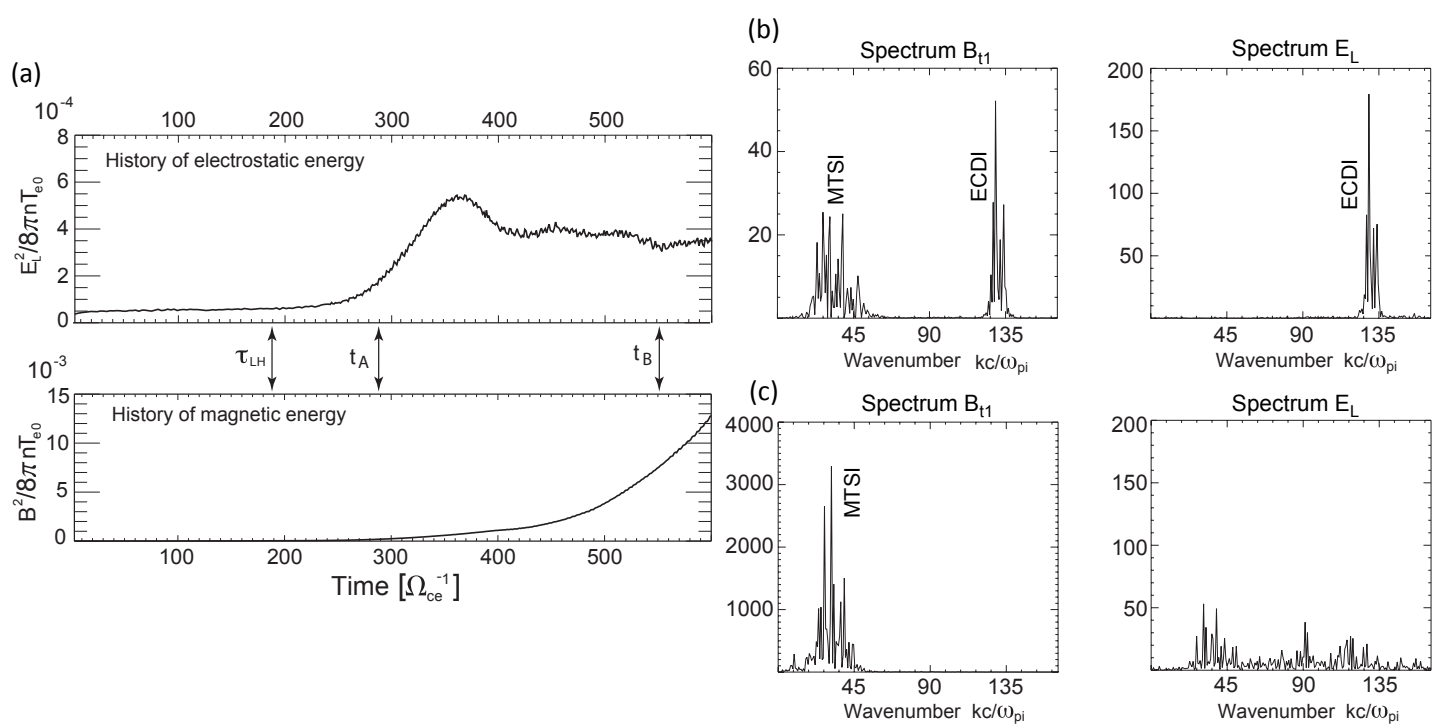

Figure 14. Quasi-perpendicular instability for $\theta=84^{\circ}$ (MTSI and ECDI). (a) Time history of the electrostatic and magnetic energy. For reference we show the lower-hybrid period $\tau_{\mathrm{LH}}$ as in Fig. 12. (b) Spectra at $t_{\mathrm{A}}=288 \Omega_{\mathrm{ce}}^{-1}$ agree with predictions from the dispersion study: wavenumbers extend in the range $20<k c / \omega_{\text {pi }}<45$ for the whistler (destabilized by the slow drift of the ion core) and $k c / \omega_{\text {pi }} \sim 130$ (viz. $\left.k \rho_{\mathrm{e}} \sim 1.44\right)$ for the extended Bernstein wave (destabilized by the fast drift of the ion beam). (c) Spectra at $t_{\mathrm{B}}=550 \Omega_{\mathrm{ce}}^{-1}$ show that the whistler has grown manyfold (compare the different amplitudes of the magnetic component $B_{t_{1}}$ at $t_{\mathrm{A}}$ and $t_{\mathrm{B}}$ ). Its electrostatic component remains modest though. The Bernstein wave has died out due to trapping of the beam ions.

using the value $c$ of Table 1 . This value is very close to the projection of the beam speed (see Table 2) along the direction of the wave normal: $\sin \left(55^{\circ}\right) \times 10 V_{\mathrm{A}}=8.2 V_{\mathrm{A}}$. It also confirms that the wave is carried by the ion beam.

\subsection{Unstable quasi-perpendicular whistlers and Bernstein waves}

Here, we set $\theta=84^{\circ}$ and run the simulation for a somewhat shorter time than in the previous case as the instability develops faster. The simulation in fact exhibits two instabilities: one at early time which has significant electrostatic energy and one at later time which is dominated by magnetic energy. Figure 14a shows the time history of the electrostatic energy $E_{\mathrm{L}}^{2} / 8 \pi$ and the magnetic energy $B^{2} / 8 \pi$, both normalized as in Sect. 5.2 by the kinetic energy of the electrons at $t=0$. Early in the run, the electrostatic energy dominates the magnetic energy. At time $\Omega_{\mathrm{ce}} t=288$, both electrostatic and magnetic energy equal $1.9 \times 10^{-4} n T_{\mathrm{e} 0}$ and $B^{2}$ crosses over $E_{\mathrm{L}}^{2}$. Thereafter, $B^{2}$ dominates $E_{\mathrm{L}}^{2}$, which reaches a maximum at $\Omega_{\mathrm{ce}} t=360$ and saturates on levels $E_{\mathrm{L}}^{2} /(8 \pi) \leq 4 \times 10^{-4} n T_{\mathrm{e} 0}$. On the other hand, $B^{2} /(8 \pi) \sim 10^{-2} n T_{\mathrm{e} 0}$ by the end of the run, namely over an order of magnitude larger and still keeps increasing.

Let us now examine the power spectra of the fields at time $t_{\mathrm{A}}=288 \Omega_{\mathrm{ce}}^{-1}$. Figure $14 \mathrm{~b}$ shows both the magnetic spectrum associated with $B_{t_{1}}$ (comparable to $B_{t_{2}}$ ) and the electrostatic spectrum of $E_{\mathrm{L}}$. The magnetic spectrum clearly exhibits two types of waves. The short wavelength type with $k c / \omega_{\mathrm{pi}} \approx 130 \pm 5$ is attributed to the ECDI and corresponds to an extension of Bernstein mode for propagation angles off $90^{\circ}$ (Muschietti and Lembège, 2013). Indeed, the dispersion analysis of Fig. $11 \mathrm{~b}$ predicts that for $\theta=84^{\circ}$ the beam can excite waves with wavenumbers in the range $1.44<$ $k \rho_{\mathrm{e}}<1.51$, or equivalently $130<k c / \omega_{\mathrm{pi}}<136$ after using Tables 1 and 2 . The waves have a strong electrostatic component visible in the spectrum of $E_{\mathrm{L}}$ and a magnetic component showing up here in the spectrum of $B_{t_{1}}$. They are destabilized by the fast drift of the ion beam versus the electrons, which is confirmed by their signature in the ion phase space (not shown). The long wavelength type with $20<k c / \omega_{\text {pi }}<45$ is due to the slow drift of the ion core versus the electrons (see Fig. 1b). Indeed, the dispersion analysis of Fig. 10 predicts that for $\theta=84^{\circ}$ the ion core excites waves with wavenumbers precisely in this range. The waves do have an electrostatic part too weak to show up in the spectrum of $E_{\mathrm{L}}$ at this early time. Being excited and carried by the ion core, they propagate in the direction opposite to the Bernstein waves.

Figure $14 \mathrm{c}$ shows the spectra of $B_{t_{1}}$ and $E_{\mathrm{L}}$ at late time $t_{\mathrm{B}}=550 \Omega_{\mathrm{ce}}^{-1}$. The quasi-perpendicular whistlers have grown tremendously, as evidenced by comparing the scales between Fig. 14b and c. Their level is now so high that their presence is visible in the phase space of the ion core (not shown). They show up equally in the spectrum of $E_{\mathrm{L}}$. At such a late time the ECDI has saturated by trapping the ion beam as explained in Muschietti and Lembège (2013) and the short-wavelength Bernstein wave has died out. 
(a)
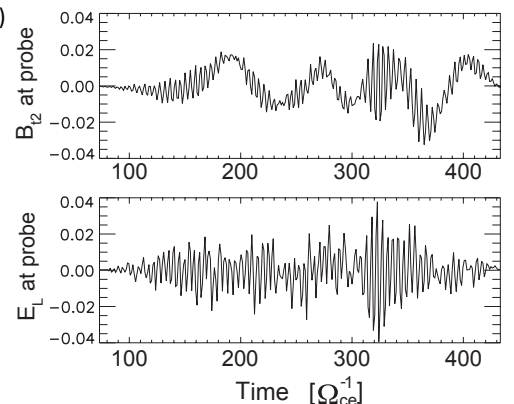

(b)

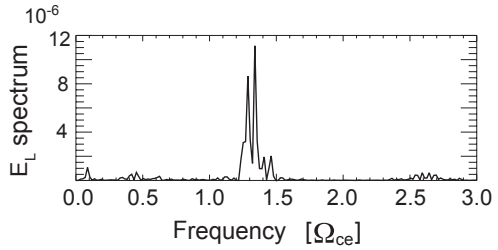

Figure 15. Quasi-perpendicular instability for $\theta=84^{\circ}$ (MTSI and ECDI) and $\beta_{\mathrm{e}}=0.22$. (a) Time variation of the magnetic component $B_{t_{2}}$ showing both the growing low-frequency whistler and the high-frequency extended Bernstein wave. The concurrent electrostatic signal $E_{\mathrm{L}}$ confirms the identification of the Bernstein wave. We note that the two waves are moving in opposite directions.

We now investigate the frequencies involved in the two types of waves in order to further the link between the simulation run and the dispersion analysis presented in Sects. 3 and 4. Probes at fixed positions record the local magnetic and electric fields versus time. Figure 15 shows a sample of such recordings. The magnetic signal, which is illustrated by $B_{t_{2}}$ in Fig. 15a, clearly exhibits a slow oscillation with period of order $\Omega_{\text {ce }} t=90$ on which a fast oscillation is superimposed; the fast oscillations appear in the electrostatic signal $E_{\mathrm{L}}$ too. The slow oscillation (period 3-4 $\omega_{\mathrm{LH}}^{-1}$ ) is due to the growing quasi-perpendicular whistler, whereas the fast oscillation (period $0.15 \omega_{\mathrm{LH}}^{-1}$ ) is attributed to the Bernstein wave. Indeed, the power spectrum of the electrostatic signal, shown in Fig. $15 \mathrm{~b}$, indicates a frequency close to $1.35 \Omega_{\mathrm{ce}}$, which is exactly the value expected for a Bernstein wave excited by the relative fast drift of the ion beam versus the electrons (see Fig. 11b).

The quasi-perpendicular whistlers are the result of an instability that has been known for a long time and is generally referred to as modified two-stream instability (MTSI) (e.g., McBride et al., 1972; Matsukiyo and Scholer, 2003). A reason this instability has attracted a lot of attention is that it has the remarkable property of requiring only a modest drift between ion and electron populations and, unlike the ion-acoustic instability, does not need a large ratio of electron to ion temperatures. For example, here one has $\left|V_{\mathrm{c}}\right|=$ $0.25 v_{\text {te }}$ and $T_{\mathrm{e}}=T_{\mathrm{c}}$. Even though the waves have a large magnetic energy, indeed much larger than the electrostatic energy as evident in Fig. 14 at time $t_{\mathrm{B}}$, their electric field is mostly electrostatic. For propagation angles close to per- pendicular (here $\theta=84^{\circ}$ ), the longitudinal component $E_{\mathrm{L}}$ completely dominates the transverse component $E_{\mathrm{t}}$, in agreement with the results of Wu et al. (1983) such as in their Fig. 7. The apparent paradox derives from the large refraction index of the excited waves. As we have seen in Figs. 10 and 14 , the wavelengths of these whistlers are such that $k c / \omega_{\mathrm{pe}} \approx 1$. Hence, the refraction index $k c / \omega \approx \omega_{\mathrm{pe}} / \omega \approx$ $\omega_{\mathrm{pe}} / \Omega_{\mathrm{ce}} \sqrt{M / m} \gg 1$. Thus, applying Faraday's law, one can write $E_{\mathrm{L}}^{2} / E_{\mathrm{t}}^{2}=(M / m)\left(E_{\mathrm{L}}^{2} / B^{2}\right)$, which shows that thanks to the large mass ratio the electric field can be mostly electrostatic $\left(\left|E_{\mathrm{L}}\right| /\left|E_{\mathrm{t}}\right| \gg 1\right)$, while simultaneously the ratio $E_{\mathrm{L}}^{2} / B^{2}$ is small.

Because a quasi-perpendicular whistler has a wavevector at a slight angle off $90^{\circ}$ to $\boldsymbol{B}_{o}$, its longitudinal electric field drives the electrons in two distinct motions: a simple parallel acceleration along $\boldsymbol{B}_{o}$ and a $E_{\mathrm{L}} \times \boldsymbol{B}_{o}$ drift in the direction $t_{1}$ defined in Fig. 2. The two associated fluctuating electron currents $j_{\|}$and $j_{\perp}$ drive the magnetic oscillation with $B_{t_{1}} \approx(4 \pi / c)\left(j_{\|} / k\right)$ and $B_{t_{2}} \approx(4 \pi / c)\left(j_{\perp} / k\right)$. As discussed by Matsukiyo and Scholer (2003), the ratio $\left|B_{t_{1}}\right| /\left|B_{t_{2}}\right|$ of the unstable waves depends upon $\beta_{\mathrm{e}}$ : the ratio is equal to 1 for very small values of $\beta_{\mathrm{e}}$ yet increases for warm plasmas. Matsukiyo and Scholer (2003) attribute this change to a decrease in the fluctuating current $j_{\perp}$ in a warm plasma. Indeed, considering that $k \rho_{\mathrm{e}}=k c / \omega_{\mathrm{pe}} \sqrt{\beta_{\mathrm{e}} / 2} \approx \sqrt{\beta_{\mathrm{e}} / 2}$ for the unstable waves, it is physically clear that, as $\beta_{\mathrm{e}}$ approaches 1 , the gyroradius becomes comparable to the wavelength for a growing part of the electron population. These electrons cease to $E_{\mathrm{L}} \times \boldsymbol{B}_{o}$ drift, whereby the current $j_{\perp}$ decreases.

The other, parallel fluctuating current $j_{\|}$is linked with the damping that comes from the Cerenkov resonance $v_{r 0}$ (defined in Eq. 4). Even for as large an angle as $84^{\circ}, v_{r 0}$ still lies on the electron distribution: a wave with $k c / \omega_{\mathrm{pe}}=1$ has $v_{r 0} \approx 2 v_{\text {te }}$ according to Fig. 6a, and an associated damping on the electrons of $\gamma \approx-0.12 \omega=-7 \Omega_{\mathrm{ci}}$ according to Fig. 5a. This damping is overcome by the destabilizing effect caused by the slow drift of the ion core versus the electrons, leading to unstable quasi-perpendicular whistlers. Our argumentation shows again that the electrons have to be treated kinetically when computing the instability's growth rate (Wu et al., 1983; Matsukiyo and Scholer, 2003). Warm electrons both cut the range of unstable wavenumbers and reduce their growth rates, as shown in Fig. 4 of Matsukiyo and Scholer (2003). Owing to this unavoidable kinetic nature of the electrons, Wu et al. (1983) renamed the instability "kinetic crossfield streaming instability". However, the moniker MTSI has stuck in the literature.

\section{Discussion}

We now further discuss the relation of the two-stream instabilities studied in this paper with work published previously. Our aim is to provide a unified context while remaining in a "linear" regime. We believe that nonlinear stages need be 
addressed with 2-D simulations due to the potential coupling between waves that propagate at various angles once they reach large amplitudes.

For perpendicular and quasi-perpendicular propagation, two different instabilities can arise. By quasi-perpendicular, we mean here a few degrees off $90^{\circ}$ : an angle sufficient to enable the electrons to run along $\boldsymbol{B}_{o}$ under the action of the wave electric field, yet modest enough to keep the wavenumber $k_{\|}$small, in case the wave experiences strong damping on the electron distribution. The first instability is also the one which occurs at the highest frequency and has the fastest growth rate: the ECDI takes its free energy from the fast drift of the reflected ion beam versus the electrons and excites waves close to the electron cyclotron frequency. The wavevectors are directed within a narrow cone about an axis perpendicular to $\boldsymbol{B}_{o}$ and along the direction of the beam toward upstream. Wavelengths are such that $k \rho_{\mathrm{e}} \sim 1$. The instability has been identified in 1-D shock simulations (Muschietti and Lembège, 2006), reported in the 2-D simulations of Matsukiyo and Scholer (2006), and studied in detail in Muschietti and Lembège (2013) for strictly perpendicular propagation. We encounter it here again for an angle $6^{\circ}$ off perpendicular as visible in Figs. 14 and 15. An important consequence of this slight angle is that ECDI acquires a magnetic component induced by the electrons' motion along $\boldsymbol{B}_{o}$ The waves have similarities to those reported by Wind and STEREO (Breneman et al., 2013; Wilson III et al., 2011).

The second instability occurs around the lower-hybrid frequency and is due to the slow drift of the ion core versus the electrons. The wavevectors are thus directed toward the shock ramp. As shown with Eq. (7) and Fig. 7c, a slow drift, on the order of the Alfvén speed, yields unstable wavevectors close to (yet distinct from) $90^{\circ}$. Wavelengths are several times the electron inertia length such that $k c / \omega_{\text {pe }} \sim 1$. This instability is known in the literature under the moniker MTSI, which stands for "modified two-stream instability". The "two-stream" denomination stems from earliest investigations based on a simple, electrostatic, and fluid dispersion relation in which the susceptibility of the electrons associated with their unimpeded motion along $\boldsymbol{B}_{o}$ reduces to $\left(\omega_{\mathrm{pe}} / \omega\right)^{2}(\cos \theta)^{2}$. This term provided one of the "stream", whereas the other was the unmagnetized ion term $\omega_{\mathrm{pi}}^{2} /\left(\omega-k V_{\mathrm{d}}\right)^{2}$. If $\theta$ is close to $90^{\circ}$ and the angular term $\cos \theta \sim \sqrt{m_{\mathrm{e}} / M_{\mathrm{i}}}$, the electrons acquire an effective mass comparable to the ions, which gives rise to a two-stream-like instability (McBride et al., 1972). On the other hand, the instability has very little similarity to a classic two-stream instability such as the Buneman instability. We have seen in Sect. 5.3 that (i) the magnetic energy is larger than the electrostatic energy and (ii) the electrons' behavior is not fluid yet kinetic in nature and provides a significant Cerenkov damping that has to be overcome by the destabilizing effect of the relative drift of the ion core/electrons. Even though those points have been noted by a few authors since the study by Wu et al. (1983), the MTSI terminology has remained in use.

The third instability generates whistlers that propagate obliquely with respect to $\boldsymbol{B}_{o}$ and have longer wavelengths. It takes its free energy from the fast drift (many times $V_{\mathrm{A}}$ ) of the reflected ion beam versus the electrons and is thus directed toward upstream. Which wavevectors are excited is the result of a competition between the destabilizing effect of the beam and the damping on the electrons due to the Cerenkov resonance. The wavelength is a fraction of the ion inertia length and the frequency a few times the lower-hybrid frequency. Specifically, with our nominal parameters, we have maximum growth for $\lambda \approx 0.3 c / \omega_{\text {pi }}$ and $\omega \approx 4-5 \omega_{\mathrm{LH}}$, while $\theta=55^{\circ}$. Let us emphasize the variability of these values. As we have seen from Fig. 9, a range of wavenumbers and angles can be excited. Generally speaking, colder electrons enable the peak growth to shift toward shorter wavelengths and more oblique propagation angles. Further, as was discussed in connection with the location marked WH-A in Fig. 7a, decreasing the beam drift increases the wavelength. The waves share many similarities to the obliquely propagating whistlers measured in detail by Polar (Hull et al., 2012).

Our present study is at the crossroads of the works of Hellinger and Mangeney (1997) and Matsukiyo and Scholer (2006). Hellinger and Mangeney (1997) carried out simulations of quasi-perpendicular shock with a 2-D hybrid code in which they observed a right-handed mode appearing to belong to the whistler branch of the fast magnetosonic. This wave propagated toward upstream obliquely to both the shock normal and the background magnetic field. The authors suggested that these whistlers were generated by the reflected ion beam, which was supported by a dispersion analysis. The wavelengths and frequencies involved were typically $\lambda \approx c / \omega_{\text {pi }}$ and $\omega \approx 30 \Omega_{\mathrm{ci}}$. On the other hand, Matsukiyo and Scholer (2006) carried out a full PIC simulation in 2-D in order to study the evolution of the MTSI in the foot of a perpendicular shock. Besides the expected quasi-perpendicular whistlers excited by the drift of the ion core, the simulation exhibited oblique whistlers that propagated in the opposite direction and were driven by the reflected ion beam. Matsukiyo and Scholer (2006) named this other instability MTSI-2, a name which was reused by Umeda et al. (2012). The wavelengths and frequencies involved were typically $\lambda \approx 6 c / \omega_{\mathrm{pe}}\left(\lambda \approx 0.14 c / \omega_{\mathrm{pi}}\right)$ and $\omega \approx 8 \omega_{\mathrm{LH}}\left(\omega \approx 350 \Omega_{\mathrm{ci}}\right)$. These wavelengths are twice shorter and those frequencies twice higher than the oblique whistlers of our paper. This is consistent with the much colder electrons Matsukiyo and Scholer (2006) used in their simulations: $\beta_{\mathrm{e}}=0.05$ instead of our nominal $\beta_{\mathrm{e}}=0.25$. Indeed, Fig. 9a shows the predictions of our dispersion analysis for cold electrons with $\beta_{\mathrm{e}}=0.06$ : the most unstable whistlers have $\lambda \approx 0.2 \mathrm{c} / \omega_{\mathrm{pi}}=6 \mathrm{c} / \omega_{\mathrm{pe}}$ and $\omega \approx 240 \Omega_{\mathrm{ci}}=8 \omega_{\mathrm{LH}}$ (precisely as in Matsukiyo and Scholer, 2006). As for the whistlers reported by Hellinger and Mangeney (1997), they have considerably longer wavelengths (by a factor of 3$)$ and lower frequencies $\left(\omega<\omega_{\mathrm{LH}}\right)$ 
than the whistlers of Matsukiyo and Scholer (2006) and the present paper. Nonetheless, we propose that Hellinger and Mangeney (1997) did indeed study the same instability in a regime with longer wavelengths. Returning to Fig. 5, we note that for all three electron temperatures the damping of the waves drops under $5 \%$ when long wavelengths such as $k c / \omega_{\mathrm{pi}} \leq 5$ are considered. This suggests that at such large scales as compared to the electron scales the role of the electrons becomes secondary, as requested for the use of a hybrid code (Hellinger and Mangeney, 1997).

Finally, we should mention that the MMS mission, with its four spacecraft, has made measurements of whistlers in the Earth's bowshock which can establish wavevector characteristics together with plasma populations down to the subion inertia scales. While a typical lower-hybrid period is $\tau_{\mathrm{LH}} \sim 100 \mathrm{~ms}$, the fluxgate magnetometer can sample waveforms in all the magnetic field components at the rate of 128 samples per second (Russell et al., 2016). Simultaneously, the Fast Plasma Investigation (Pollock et al., 2016) can provide electron distributions down to $30 \mathrm{~ms}$ and ion distributions down to $150 \mathrm{~ms}$. These cadences are certainly high enough to study the role of the ions with respect to the lowerhybrid whistlers and the effects the latter have on the electrons.

\section{Conclusion}

In this study we have examined the wave activity that can possibly develop in the foot of quasi-perpendicular shocks, as it arises from the relative drifts across the background magnetic field $\boldsymbol{B}_{o}$ of three particle populations: incoming ions, reflected ions, and electrons. Our main goal was to consider the role of different wave propagation angles with respect to $\boldsymbol{B}_{o}$ and compare the corresponding two-stream instabilities. Three main types of instabilities and correspondingly excited waves are identified:
- Generalized Bernstein waves with wavelengths close to the electron gyroradius which propagate toward upstream at angles within a few degrees off $90^{\circ}$ to $\boldsymbol{B}_{o}$. Frequencies are close to the electron cyclotron. Their free energy is provided by the "fast" relative drift between the reflected ions/electrons.

- Quasi-perpendicular whistlers with wavelength covering several times the electron inertia length (such that $\left.k c / \omega_{\text {pe }} \sim 1\right)$ which propagate downstream toward the ramp at angles larger than $80^{\circ}$ to $\boldsymbol{B}_{o}$. Frequencies are close to the lower hybrid. Their free energy is provided by the "slow" relative drift between the incoming ions/electrons.

- Oblique whistlers with wavelengths close to the ion inertia length which propagate toward upstream at angles about $50^{\circ}$ to $\boldsymbol{B}_{o}$. Frequencies are a few times the lower hybrid. Their free energy is provided by the "fast" relative drift between the reflected ions/electrons.

Data availability. Access to the raw data may be provided upon reasonable request to the authors. 


\section{Appendix A: The dielectric tensor}

The geometry used is displayed in Fig. 2: the background magnetic field $\boldsymbol{B}_{o}$ points in the $\hat{z}$ direction and the wave vector lies in the plane containing $\underline{\hat{x}}$ and $\hat{z}$, where it makes an angle $\theta$ to $\hat{z}$. For convenience in this appendix, we adopt the notation that indicates a one-dimensional array by one underline and a two-dimensional array by two underlines.

The dielectric tensor $\underline{\underline{\epsilon}}$ combines with the wave propagation terms to form the wave equation for the electric field

$$
\left[\underline{\underline{\epsilon}}-\left(\frac{k c}{\omega}\right)^{2} \underline{\underline{I}} \mathrm{~T}\right] \cdot \underline{E}=0,
$$

where $\underline{\underline{I}}_{T} \equiv \underline{\underline{I}}-\underline{k} \underline{k} / k^{2}$ with $\underline{\underline{I}}$ the unit tensor and $\underline{\underline{I}}_{\mathrm{T}}$ the transverse projection tensor (see, e.g., Ichimaru, 1973).

When the plasma is made of various populations, it is convenient to write the dielectric tensor as

$\underline{\underline{\epsilon}}=\underline{\underline{I}}+\underline{\underline{Q}}$,

where the contributions of the various populations are added into $\underline{Q}$. If a population is magnetized, then its contributions satisfy the symmetries: $Q_{y x}=-Q_{x y}, Q_{y z}=-Q_{z y}$, and $Q_{z x}=+Q_{x z}$. If it is considered as unmagnetized, then one has $Q_{y x}=Q_{x y}=0$ and $Q_{y z}=Q_{z y}=0$.

In our case, we are interested in waves with frequencies ranging from the lower hybrid up to the electron cyclotron. Therefore, the ions will be unmagnetized but the electrons magnetized.

For the ions' contribution we have four integrals of the following type to evaluate:

$\mathcal{I}_{\alpha \beta}=\int \mathrm{d} v_{x} \mathrm{~d} v_{y} \mathrm{~d} v_{z} \frac{v_{\alpha} v_{\beta}}{\underline{\boldsymbol{k}} \cdot \underline{v}-\omega} \underline{\boldsymbol{k}} \cdot \frac{\partial F}{\partial \underline{v}}$.

The ion distribution is modeled by a Maxwellian drifting perpendicularly to $\underline{\boldsymbol{B}}_{0}$. Since the maximum growth rate is obtained when the wavevector $\underline{k}$ lies within the plane defined by $\underline{\boldsymbol{B}}_{o}$ and the drift $\underline{V}_{\mathrm{d}}$, one chooses $\underline{V}_{\mathrm{d}}=\left(V_{x}, 0,0\right)$ and $\underline{\boldsymbol{k}}=\left(k_{x}, 0, k_{z}\right)$. After some algebra, we can express the integrals in terms of the plasma dispersion function $Z$ :

$Z(\zeta) \equiv \frac{1}{\pi^{1 / 2}} \int_{-\infty}^{+\infty} \mathrm{d} t \frac{e^{-t^{2}}}{t-\zeta}$

Elements of the tensor $\underline{\underline{Q}}$, where the argument $\zeta=(\omega / k-$ $\left.V_{x} \sin \theta\right) / \sqrt{2} v_{\mathrm{ti}}$, are given by

$$
\begin{aligned}
Q_{x x} & =\frac{\omega_{\mathrm{pi}}^{2}}{\omega^{2}}\left\{\cos ^{2} \theta \zeta Z(\zeta)-\left(\frac{V_{x}}{\sqrt{2} \mathrm{v}_{\mathrm{ti}}}+\zeta \sin \theta\right)^{2} Z^{\prime}(\zeta)\right\}, \\
Q_{x z} & =-\frac{\omega_{\mathrm{pi}}^{2}}{\omega^{2}} \cos \theta\left\{\sin \theta \zeta\left[Z(\zeta)+\zeta Z^{\prime}(\zeta)\right]+\frac{V_{x}}{\sqrt{2} v_{\mathrm{ti}}} \zeta Z^{\prime}(\zeta)\right\},
\end{aligned}
$$

$$
\begin{aligned}
& Q_{y y}=-\frac{\omega_{\mathrm{pi}}^{2}}{\omega^{2}}\left[1+\frac{1}{2} Z^{\prime}(\zeta)\right], \\
& Q_{z z}=\frac{\omega_{\mathrm{pi}}^{2}}{\omega^{2}}\left[\sin ^{2} \theta \zeta Z(\zeta)-\cos ^{2} \theta \zeta^{2} Z^{\prime}(\zeta)\right] .
\end{aligned}
$$

We can perform a few checks on those expressions. For example, if $\theta=\pi / 2$, then one can verify that $I_{\alpha \beta}+Q_{\alpha \beta}$ reduces to the usual 1-D expressions for longitudinal and transverse dielectric

$$
\begin{aligned}
& 1+Q_{x x}=1-\frac{\omega_{\mathrm{pi}}^{2}}{2 k^{2} v_{\mathrm{ti}}^{2}} Z^{\prime}(\zeta), \\
& 1+Q_{y y}=1+Q_{z z}=1+\frac{\omega_{\mathrm{pi}}^{2}}{\omega^{2}} \zeta Z(\zeta) .
\end{aligned}
$$

The electron distribution is modeled by an isotropic Maxwellian without drift. However, the electrons being magnetized, their contributions to the dielectric tensor are made of the usual sums combining modified Bessel functions and plasma dispersion function. The Bessel functions $I_{n}$ of order $n$ have $\eta \equiv\left(k \sin \theta v_{\text {te }} / \Omega_{\text {ce }}\right)^{2}$ for argument, while the $Z$ function has the parallel phase velocity shifted according to the $n$th harmonic for argument,

$\zeta_{n} \equiv \frac{\omega+n \Omega_{\mathrm{ce}}}{\sqrt{2} k \cos \theta v_{\mathrm{te}}}$.

Elements of the tensor $\underline{\underline{Q}}$ associated with the electrons are listed here:

$$
\begin{aligned}
& Q_{x x}=\frac{\omega_{\mathrm{pe}}^{2}}{\omega^{2}} \frac{1}{\eta} \sum_{n=-\infty}^{n=+\infty} n^{2} I_{n} e^{-\eta} \zeta_{0} Z\left(\zeta_{n}\right), \\
& Q_{x y}=-i \frac{\omega_{\mathrm{pe}}^{2}}{\omega^{2}} \sum_{n=-\infty}^{n=+\infty} n\left(I_{n}-I_{n}^{\prime}\right) e^{-\eta} \zeta_{0} Z\left(\zeta_{n}\right), \\
& Q_{x z}=\frac{4 \omega_{\mathrm{pe}}^{2} \Omega_{\mathrm{ce}}}{\omega k^{2} v_{\mathrm{te}}^{2} \sin 2 \theta} \sum_{n=-\infty}^{n=+\infty} n I_{n} e^{-\eta} Z^{\prime}\left(\zeta_{n}\right), \\
& Q_{y y}=\frac{\omega_{\mathrm{pe}}^{2}}{\omega^{2}} \frac{1}{\eta} \sum_{n=-\infty}^{n=+\infty}\left[n^{2} I_{n}+2 \eta^{2}\left(I_{n}-I_{n}^{\prime}\right)\right] e^{-\eta} \zeta_{0} Z\left(\zeta_{n}\right) \text {, } \\
& Q_{y z}=-i \frac{\omega_{\mathrm{pe}}^{2} \tan \theta}{2 \omega \Omega_{\mathrm{ce}}} \sum_{n=-\infty}^{n=+\infty}\left(I_{n}-I_{n}^{\prime}\right) e^{-\eta} Z^{\prime}\left(\zeta_{n}\right), \\
& Q_{z z}=-\frac{\omega_{\mathrm{pe}}^{2}}{\omega^{2}} \sum_{n=-\infty}^{n=+\infty} I_{n} e^{-\eta} \zeta_{n} \zeta_{0} Z^{\prime}\left(\zeta_{n}\right)
\end{aligned}
$$

The other elements are found via the symmetries already mentioned for magnetized species. 


\section{Appendix B: Parallel electric field}

Our purpose here is to assess how the parallel component of the electric field $\boldsymbol{E}_{z}$ varies with the direction of the whistler wave. For the sake of tractability we will assume the cold plasma model, for which the terms $Q_{\alpha \beta}$ of the dielectric tensor simplify considerably. One has (see, e.g., Swanson, 2003)

$$
\begin{aligned}
& Q_{x x}=Q_{y y}=-\frac{\omega_{\mathrm{pe}}^{2}}{\omega^{2}-\Omega_{\mathrm{ce}}^{2}}-\frac{\omega_{\mathrm{pi}}^{2}}{\omega^{2}-\Omega_{\mathrm{ci}}^{2}}, \\
& Q_{y x}=-Q_{x y}=-i \frac{\Omega_{\mathrm{ce}}}{\omega} \frac{\omega_{\mathrm{pe}}^{2}}{\omega^{2}-\Omega_{\mathrm{ce}}^{2}}+i \frac{\Omega_{\mathrm{ci}}}{\omega} \frac{\omega_{\mathrm{pi}}^{2}}{\omega^{2}-\Omega_{\mathrm{ci}}^{2}}, \\
& Q_{z z}=-\frac{\omega_{\mathrm{pe}}^{2}}{\omega^{2}}-\frac{\omega_{\mathrm{pi}}^{2}}{\omega^{2}} .
\end{aligned}
$$

All other terms are null.

The wave Eq. (A1) includes a $3 \times 3$ matrix, which leads to three scalar equations. For the cold model, the last two scalar equations read

$$
\begin{aligned}
& Q_{y x} \boldsymbol{E}_{x}+\left[Q_{y y}+1-\left(\frac{k c}{\omega}\right)^{2}\right] \boldsymbol{E}_{y}=0, \\
& \left(\frac{k c}{\omega}\right)^{2} \cos \theta \sin \theta \boldsymbol{E}_{x}+\left[Q_{z z}+1-\left(\frac{k c}{\omega}\right)^{2} \sin ^{2} \theta\right] \boldsymbol{E}_{z}=0 .
\end{aligned}
$$

After eliminating $\boldsymbol{E}_{x}$, one can express the parallel component of the electric field $\boldsymbol{E}_{z}$ as a ratio to $\boldsymbol{E}_{y}$, viz. the component that is both transverse and perpendicular:

$$
\frac{\boldsymbol{E}_{z}}{\boldsymbol{E}_{y}}=\left(\frac{k c}{\omega}\right)^{2} \cos \theta \sin \theta \frac{Q_{y y}+1-\left(\frac{k c}{\omega}\right)^{2}}{Q_{y x}\left[Q_{z z}+1-\left(\frac{k c}{\omega}\right)^{2} \sin ^{2} \theta\right]}
$$

Note that $Q_{y x}$ is imaginary, which means that the phases of $\boldsymbol{E}_{z}$ and $\boldsymbol{E}_{y}$ are in quadrature. It is also noteworthy that the parallel component vanishes both for parallel propagation, $\theta \rightarrow 0$, and for perpendicular propagation, $\theta \rightarrow \pi / 2$. In the latter case, one has an extraordinary wave, where the polarization is linear with the electric field oscillating perpendicularly to $\boldsymbol{B}_{o}$. In the former case, one has a circularly polarized transverse wave with the electric field rotating in the $[x, y]$ plane.
In order to extract further information from Eq. (B6), we need to introduce approximations to $Q_{\alpha, \beta}$ which result from the ordering of frequencies for the oblique whistlers: $\Omega_{\mathrm{ci}} \ll$ $\omega \ll \Omega_{\text {ce }} \ll \omega_{\text {pe }}$. We then have

$$
\begin{aligned}
Q_{y y} & \approx\left(\frac{\omega_{\mathrm{pe}}}{\Omega_{\mathrm{ce}}}\right)^{2}\left(1-\frac{\omega_{\mathrm{LH}}^{2}}{\omega^{2}}\right) \quad Q_{y x} \approx i \frac{\omega_{\mathrm{pe}}^{2}}{\Omega_{\mathrm{ce}} \omega} \\
Q_{z z} & \approx-\frac{\omega_{\mathrm{pe}}^{2}}{\omega^{2}},
\end{aligned}
$$

which are substituted in Eq. (B6) to yield

$$
\frac{i \boldsymbol{E}_{z}}{\boldsymbol{E}_{y}}=\frac{m}{M}\left(\frac{k c}{\omega_{\mathrm{pi}}}\right)^{4} \frac{\Omega_{\mathrm{ci}}}{\omega} \cos \theta \sin \theta \frac{1-\left(\frac{\omega_{\mathrm{pi}}}{k c}\right)^{2}\left(\frac{\omega^{2}}{\omega_{\mathrm{LH}}^{2}}-1\right)}{1+\left(\frac{k c}{\omega_{\mathrm{pe}}}\right)^{2} \sin ^{2} \theta} .
$$

This equation still has an implicit angular dependence via $\omega$ in the denominator. Let us define the function of order unity

$$
U(k) \equiv \frac{1-\left(\frac{\omega_{\mathrm{pi}}}{k c}\right)^{2}\left(\frac{\omega^{2}}{\omega_{\mathrm{LH}}^{2}}-1\right)}{1+\left(\frac{k c}{\omega_{\mathrm{pe}}}\right)^{2} \sin ^{2} \theta}
$$

and use Eq. (1) to eliminate the frequency dependence in the denominator of Eq. (B7). We obtain

$$
\begin{aligned}
\frac{i \boldsymbol{E}_{z}}{\boldsymbol{E}_{y}} & \approx \frac{m}{M}\left(\frac{k c}{\omega_{\mathrm{pi}}}\right)^{3} \\
& \times \frac{\left[1+\left(k c / \omega_{\mathrm{pe}}\right)^{2}\right] \cos \theta \sin \theta}{\left[1+\left(k c / \omega_{\mathrm{pe}}\right)^{2}+\left(k c / \omega_{\mathrm{pi}}\right)^{2} \cos ^{2} \theta\right]^{1 / 2}} U(k) .
\end{aligned}
$$

Two remarks are in order. First, due the mass ratio, one can expect the parallel electric field to remain modest, i.e., $\left|\boldsymbol{E}_{z}\right|<\left|\boldsymbol{E}_{y}\right|$ for wavenumbers less than the electron inertia length. For example, if $k c / \omega_{\mathrm{pi}}=15$ and $M / m=900$ one has $\left|\boldsymbol{E}_{z}\right| /\left|\boldsymbol{E}_{y}\right| \lesssim 0.25$. Second, the angular dependence maximizes about $\theta \sim 70^{\circ}$ and this angle gets larger as the wavenumber increases. 
Competing interests. The authors declare that they have no conflict of interest.

Acknowledgements. Laurent Muschietti gratefully acknowledges beneficial conversations with Art Hull and also thanks the LATMOS, where he has worked at in France, for the hospitality and the access to computing facilities while some of this study was done. The PIC simulations were performed on ADA, a machine of the supercomputer center "IDRIS", institute of the CNRS (http: //www.idris.fr) located at Orsay (near Paris). This work has been partly supported by the NASA grant NNX10A109G to UC Berkeley and partly by CNES (French Space Center) and by the program "Investissements d'avenir" under the reference ANR MACH (ANR14-CE33-0019-06).

The topical editor, Christopher Owen, thanks two anonymous referees for help in evaluating this paper.

\section{References}

Breneman, A. W., Cattell, C. A., Kersten, K., Paradise, A., Schreiner, S., and Kellogg, P. J.: Stereo and Wind observations of intense cyclotron harmonic waves at the Earth's bow shock and inside the magnetosheath, J. Geophys. Res., 118, 7654-7664, https://doi.org/10.1002/2013JA019372, 2013.

Dimmock, A. P., Balikhin, M. A., Walker, S. N., and Pope, S. A.: Dispersion of low frequency plasma waves upstream of the quasi-perpendicular terrestrial bow shock, Ann. Geophys., 31, 1387-1395, https://doi.org/10.5194/angeo-31-1387-2013, 2013.

Hellinger, P. and Mangeney, A.: Upstream whistlers generated by protons reflected from a quasi-perpendicular shock, J. Geophys. Res., 102, 9809-9819, 1997.

Hull, A. J., Muschietti, L., Oka, M., Larson, D. E., Mozer, F. S., Chaston, C. C., Bonnell, J. W., and Hospodarsky, G. B.: Multi-scale whistler waves within Earth's perpendicular bow shock, J. Geophys. Res., 117, A12104, https://doi.org/10.1029/2012JA017870, 2012.

Ichimaru, S.: Basic Principles of Plasma Physics, A Statistical Approach, Series in Frontiers in Physics, W.A. Benjamin, Reading, Massuchusetts, 1973.

Krasnoselskikh, V. V., Balikhin, M. A., Alleyne, H., Klimov, S. I., Mier-Jedrzejowicz, W. A. C., Pardaens, A. K., Petrukovich, A., Southwood, D. J., Vinogradova, T., and Woolliscroft, L. J. C.: On the nature flow frequency turbulence in the foot of strong quasiperpendicular shocks, Adv. Space Res., 11, 15-18, 1991.

Lembege, B.: Antenna radiation pattern of cyclotron harmonic waves in a hot magnetoplasma, Radio Sci., 14, 487-502, 1979a.

Lembege, B.: Method for predicting the properties of cyclotron harmonics waves from perpendicular dispersion relation, J. Plasma Phys., 22, 231-244, 1979b.

Lembege, B. and Gonfalone, A.: Cyclotron harmonic waves propagation in a large magnetoplasma, Plasma Phys., 20, 879-889, 1978.
Matsukiyo, S. and Scholer, M.: Modified two-stream instability in the foot of high Mach number quasiperpendicular shocks, J. Geophys. Res., 108, 1459-1469, https://doi.org/10.1029/2003JA010080, 2003.

Matsukiyo, S. and Scholer, M.: On microinstabilities in the foot of high Mach number perpendicular shocks, J. Geophys. Res., 111, A06104, https://doi.org/10.1029/2005JA011409, 2006.

McBride, J. B., Ott, E., Boris, J. P., and Orens, J. H.: Theory and simulation of turbulent heating by the modified two-stream instability, Phys. Fluids, 15, 2367-2383, 1972.

Muschietti, L. and Lembège, B.: Electron cyclotron microinstability in the foot of a perpendicular shock: a selfconsistent PIC simulation, Adv. Space Res., 37, 483-493, https://doi.org/10.1016/j.asr.2005.03.077, 2006.

Muschietti, L. and Lembège, B.: Microturbulence in the electron cyclotron frequency range at perpendicular supercritical shocks, J. Geophys. Res., 118, 2267-2285, https://doi.org/10.1002/jgra.50224, 2013.

Pollock, C., Moore, T., Jacques, A., et al.: Fast plasma investigation for magnetospheric multiscale, Space Sci. Rev., 199, 331-406, https://doi.org/10.1007/s11214-016-0245-4, 2016.

Rodriguez, P. and Gurnett, D. A.: Electrostatic and electromagnetic turbulence associated with Earth's bow shock, J. Geophys. Res., 80, 19-31, 1975.

Russell, C. T., Anderson, B. J., Baumjohann, W., Bromund, K. R., Dearborn, D., Fischer, D., Le, G., Leinweber, H. K., Leneman, D., Magnes, W., Means, J. D., Moldwin, M. B., Nakamura, R., Pierce, D., Plaschke, F., Rowe, K. M., Slavin, J. A., Strangeway, R. J., Torbert, R., Hagen, C., Jernej, I., Valavanoglou, A., and Richter, I.: The magnetospheric multiscale magnetometers, Space Sci. Rev., 199, 189-256, https://doi.org/10.1007/s11214014-0057-3, 2016.

Stringer, T. E.: Low-frequency waves in an unbounded plasma. Plasma Phys., 5, 89, 1963.

Sundkvist, D., Krasnoselskikh, V., Bale, S. D., Schwartz, S. J., Soucek, J., and Mozer, F.: Dispersive nature of high mach number collisionless plasma shocks: Poynting flux of oblique whistler waves, Phys. Rev. Lett., 108, 025002, https://doi.org/10.1103/PhysRevLett.108.025002, 2012.

Swanson, D. G.: Plasma waves, 2nd Edn., Series in plasma Physics, Institute of Physics Publishing, London, 2003.

Thiel, J. and Lembège, B.: Electrostatic electron cyclotron radiation from a point source, Phys. Fluids, 25, 551-565, 1982.

Tidman, D. A. and Krall, N. A.: Shock Waves in Collisionless Plasmas, Wiley-Interscience, New York, 1971.

Umeda, T., Kidani, Y., Matsukiyo, S., and Yamazaki, R.: Modified two-stream instability at perpendicular collision less shocks: Full particle simulations, J. Geophys. Res., 117, A03206, https://doi.org/10.1029/2011JA017182, 2012.

Wilson III, L. B., Cattell, C. A., Kellogg, P. J., Goetz, K., Kersten, K., Kasper, J. C., Szabo, A., and Wilber, M.: Large-amplitude electrostatic waves observed at a supercritical interplanetary shock, J. Geophs. Res., 115, A12104, https://doi.org/10.1029/2010JA015332, 2010.

Winske, D., Tanaka, M., Wu, C. S., and Quest, K. B.: Plasma heating at collisionless shocks due to the kinetic cross-field streaming instability, J. Geophys. Res., 90, 123-136, 1985. 
Wu, C. S., Zhou, Y. M., Tsai, S. T., Guo, S. C., Winske, D., and Papadopoulos, K.: A kinetic cross-field streaming instability, Phys. Fluids, 26, 1259-1267, 1983.
Wu, C. S., Winske, D., Zhou, Y. M., Tsai, S. T., Rodriguez, P., Tanaka, M., Papadopoulos, K., Akimoto, K., Lin, C. S., Leroy, M. M., and Goodrich, C. C.: Microinstabilities associated with a high Mach number, perpendicular bow shock, Space Sci. Rev., 37, 63-109, 1984. 